\title{
Output Feedback Adaptive Dynamic Surface Sliding-Mode Control for Quadrotor UAVs with Tracking Error Constraints
}

\author{
Guoqiang Zhu, ${ }^{1,2}$ Sen Wang, ${ }^{1,2}$ Lingfang Sun $\mathbb{D}^{1,2}$ Weichun Ge, ${ }^{3}$ and Xiuyu Zhang $\mathbb{C}^{1,2}$ \\ ${ }^{1}$ School of Automation Engineering, Northeast Electric Power University, Jilin, China \\ ${ }^{2}$ Jilin Province International Research Center of Precision Drive and Intelligent Control, Jilin, China \\ ${ }^{3}$ State Grid Liaoning Electric Power Supply Co., Ltd., Shenyang, China \\ Correspondence should be addressed to Xiuyu Zhang; zhangxiuyu80@163.com
}

Received 11 April 2020; Accepted 22 May 2020; Published 15 June 2020

Guest Editor: Ning Wang

Copyright (c) 2020 Guoqiang Zhu et al. This is an open access article distributed under the Creative Commons Attribution License, which permits unrestricted use, distribution, and reproduction in any medium, provided the original work is properly cited.

In this paper, a fuzzy adaptive output feedback dynamic surface sliding-mode control scheme is presented for a class of quadrotor unmanned aerial vehicles (UAVs). The framework of the controller design process is divided into two stages: the attitude control process and the position control process. The main features of this work are (1) a nonlinear observer is employed to predict the motion velocities of the quadrotor UAV; therefore, only the position signals are needed for the position tracking controller design; (2) by using the minimum learning technology, there is only one parameter which needs to be updated online at each design step and the computational burden can be greatly reduced; (3) a performance function is introduced to transform the tracking error into a new variable which can make the tracking error of the system satisfy the prescribed performance indicators; (4) the slidingmode surface is introduced in the process of the controller design, and the robustness of the system is improved. Stability analysis proved that all signals of the closed-loop system are uniformly ultimately bounded. The results of the hardware-in-the-loop simulation validate the effectiveness of the proposed control scheme.

\section{Introduction}

Quadrotor unmanned aerial vehicles (UAVs), as a new product in the field of small UAVs, have become a research hotspot among research and scholars all over the word [1-5]. The main advantages of quadrotor UAVs, such as flying in any direction, take off and land vertically, and hover at an ideal attitude, make the quadrotor UAVs widely used in more important fields, such as providing medical assistance, transporting special resources, disaster monitoring, and agricultural mapping. However, quadrotor UAVs are a complex physical system with the following characteristics, such as multivariate, nonlinearity, underactuating, and strong coupling, which make it very difficult to design an effective adaptive robust flight controller.

In the recent years, various effective control techniques have been developed for quadrotor UAVs to achieve stabilized or automatic flight, such as adaptive PID linear quadratic regulator (LQR) control [2, 6, 7], LMI-based linear control $[8,9]$, and $H_{\infty}$ control $[10,11]$.
With the development of intelligence control theory, different kinds of advanced nonlinear control methods, which combine the linear control methods with intelligence control theory, such as feedback linearization control [12], model predictive control [13, 14], adaptive backstepping control $[15,16]$, adaptive sliding-mode control (SMC) [17-20], fault-tolerant control [21], dynamic surface control (DSC) [22-25], and adaptive fuzzy control $[26,27]$, have been proposed to achieve attitude and position trajectory tracking performance of quadrotor UAVs. In [28], a novel neural network-based output feedback controller is developed for a group of quadrotor UAVs. In [29], the prescribed performance backstepping dynamic surface control (DSC) scheme is proposed to solve the problem of trajectory tracking control for a quadrotor UAV with control input saturation. In [5], a fuzzy-based compound quantized control strategy is applied to the Quanser Qball-X4 quadrotor experimental platform, which achieved precise position control and tracking performance. 
Among the above control schemes, the backstepping strategy has been widely used in the controller design for nonlinear systems. For instance, in [30], the trajectory tracking controller based on the backstepping approach was developed for the quadrotor model, while the PD control was used to attenuate the effects caused by system uncertainties. In [16], a nonlinear disturbance observer-based backstepping control method has been proposed to address the problem of loss of actuators' effectiveness. However, one drawback of backstepping is the "explosion of complexity" caused by the recurrent derivation of the virtual control law in each design step. To deal with this problem, the DSC control method has been proposed for a class of nonlinear systems, by introducing a first-order low-pass filter in each design step, and the shortcoming was overcome [22, 31-33].

An effective way to deal with the uncertainty of system parameters and unmodeled dynamics is to design an adaptive controller using the general approximation ability of the fuzzy logic system (FLS) and neural networks (NNs) [34-36]. The number of adaptive laws which depends on the fuzzy rules or the NN weights will be significantly increased as the number of fuzzy rules or the NN weights increase. To overcome this problem, a new method by estimating the norm rather than each item of the weight vector was proposed in $[37,38]$. Thus, the number of adaptive laws is reduced significantly. Actually, the quadrotor UAVs are not only time-varying coupled and nonlinear systems, but also suffer from perturbations such as payload variations and nonlinear friction. Therefore, it is necessary to design a controller with adaptive capability, fast convergence, and robust performance for the quadrotor UAVs.

As a widely used nonlinear control algorithm, the sliding-mode control (SMC) is known for its excellent performance properties for complex high-order nonlinear systems in the presence of uncertain conditions $[39,40]$. In [18], the SMC trajectory tracking controller was proposed for quadrotor UAVs by considering the wind perturbations and external disturbance components. In [19], a hierarchical control strategy based on the double-loop integral slidingmode controller was designed for the position and attitude tracking of quadrotor UAVs with sustained disturbances and parameter uncertainties. Most of the existing literature focuses on using the SMC method to solve the attitude control of quadrotor UAVs instead of the position trajectory tracking control design because the transformed dynamic equation has a preferred form for the attitude control.

However, a major constraint in the controller design of quadrotor UAVs is that all the state variables of the system are required to be measurable. But in practical application, under some unpredictable factors, it will cause the measurement sensor to fail. [41-45]. In [41], an adaptive output feedback control scheme has been proposed for a class of uncertain SISO nonlinear systems under the constraint that only the system output can be obtained. In [43], a fuzzy state observer-based control method is designed for an uncertain MIMO nonlinear system, and by using the state observer, the problem of state immeasurability has been solved. Traditionally, the tracking performance in adaptive control schemes has been confined to ensure that the tracking error converges to a residual set, the size of which is determined by the explicit design parameters and some unknown bounded terms. The upper bounds of the tracking error are difficult to calculate, so it is a very practical work to make the prior selection of the tracking performance satisfy certain steady state behavior. In [46, 47], a prescribed performance control scheme has been proposed for a class of nonlinear systems, and by constructing a prescribed performance function, the tracking error of the system was transformed into a new variable to ensure that the convergence rate was no less than a prespecified value, and the steady-state error remains within the prescribed range. However, limited attention has been paid to this issue for the controller design of quadrotor UAVs.

Inspired by the aforementioned discussions, an adaptive output feedback dynamic surface sliding-mode control for a class of quadrotor UAV system is presented where the fuzzy approximators are used to approximate the unknown items of the system. The main contributions of the proposed control scheme are as follows:

Firstly, to our best knowledge, this is the first time to use the dynamic surface control techniques with the slidingmode method to design and test the robust controller of quadrotor UAVs in the platform of hardware-in-the-loop simulation, leading to a greatly simplified structure of the controller and improved robustness of the system.

Secondly, by introducing performance and error transformed functions in the controller, the tracking error of the quadrotor UAVs is transformed into a new error constraint variable which can ensure the prescribed transient performance of the system.

Thirdly, by estimating the norm of the FLS weights instead of estimating each variables of the weight vector, there is only one parameter needed to be updated at each step. Thus, the computing time is reduced.

Finally, the nonlinear state observer is introduced to predict the unmeasurable state of the quadrotor such as the angular velocity state of the quadrotor. Then, only the measurable attitude and position information are required in the implementation of the controller of the quadrotor.

The rest of this paper is organized as follows. In Section 2, problem statement and preliminaries including the mathematical model of the quadrotor, fuzzy logic systems (FLSs), and performance function are introduced. The process of the controller design and analysis of stability are given in Sections 3 and 4, respectively. Section 5 shows the results of the hardware-in-the-loop simulation to validate the effectiveness of the proposed control scheme.

\section{Problem Statement and Preliminaries}

2.1. Dynamic Model of Quadrotor UAVs. The schematic configuration of the quadrotor in this paper is shown in Figure 1. The basic movements are vertical movement, front and back movement, left and right movement, pitch rotation, roll rotation, and yaw rotation. On changing the rotor speed altogether with the same quantity, the lift forces will change, in this case, affecting the attitude of the vehicle. The complicated motions of a quadrotor can be divided into two 
typical parts, and each part represents a subsystem with coupled terms. The first part is associated with the translational positions, and the second part is associated with the rotational angles. And in this section, we will deduce the mathematical model of a quadrotor UAV, including navigation equations and moment equations.

Define $\Lambda=[\phi, \theta, \psi]^{T} \in R^{3}$ and $w=[p, q, r]^{T}$, where $\phi, \theta$, and $\psi$ represent the roll angle, pitch angle, and yaw angle with respect to the inertia frame and $p, q$, and $r$ denote the angular velocity of the roll, pitch, and yaw with respect to the body-fixed frame. Let $R_{\mathrm{BG}}$ denote the transformation matrix between the inertia frame and the body-fixed frame using Euler-Lagrange formulation, which can be expressed as

$$
R_{\mathrm{BG}}=\left[\begin{array}{ccc}
C_{\psi} C_{\theta} & C_{\psi} S_{\theta} S_{\phi}-S_{\psi} C_{\phi} & C_{\psi} S_{\theta} C_{\phi}+S_{\psi} S_{\phi} \\
S_{\psi} C_{\theta} & S_{\psi} S_{\theta} S_{\phi}+C_{\psi} C_{\phi} & S_{\psi} S_{\theta} C_{\phi}-C_{\psi} S_{\phi} \\
-S_{\theta} & S_{\phi} C_{\theta} & C_{\phi} C_{\theta}
\end{array}\right],
$$

where $S_{(\cdot)}$ and $C_{(\cdot)}$ denote the $\sin (\cdot)$ and $\cos (\cdot)$, respectively.

Let $P=[x, y, z]^{T} \in R^{3}$ denote the position with respect to the inertia frame. According to Newton's second law, the relationship between combined force $F_{G}$ and acceleration in the ground coordinate is

$$
F_{G}=\left[\begin{array}{c}
F_{x} \\
F_{y} \\
F_{z}
\end{array}\right]=\frac{\mathrm{d}}{\mathrm{d} t}(m V)=m\left[\begin{array}{l}
\ddot{x} \\
\ddot{y} \\
\ddot{z}
\end{array}\right],
$$

and we can get the translational dynamic equations of the quadrotor

$$
\left[\begin{array}{c}
\ddot{x} \\
\ddot{y} \\
\ddot{z}
\end{array}\right]=\frac{1}{m} F_{G}=\frac{1}{m}\left(R_{\mathrm{BG}}\left[\begin{array}{c}
0 \\
0 \\
U_{z}
\end{array}\right]-\left[\begin{array}{c}
0 \\
0 \\
m g
\end{array}\right]-\left[\begin{array}{c}
D_{x} \\
D_{y} \\
D_{z}
\end{array}\right]\right),
$$

where $D_{x}=d_{x} \dot{x}, D_{y}=d_{y} \dot{y}$, and $D_{z}=d_{z} \dot{z}, \quad$ in $\quad$ which $d_{x}, d_{y}$, and $d_{z}$ are the air drag coefficients; $U_{z}=k\left(\Omega_{1}^{2}+\Omega_{2}^{2}+\Omega_{3}^{2}+\Omega_{4}^{2}\right)$ is the lift force generated by the rotors with respect to the body coordinate system, in which $\Omega_{i}, i=1, \ldots, 4$ denote the rotary speed of the front, right, rear, and left rotors and $k$ is the lift coefficient of the rotor.

According to the kinetic equation, the relationship between $\Lambda$ and $w$ can be described as

$$
\dot{\Lambda}=Q(\Lambda) w=\left[\begin{array}{ccc}
1 & T_{\theta} S_{\phi} & T_{\theta} C_{\phi} \\
0 & C_{\phi} & -S_{\phi} \\
0 & \frac{S_{\phi}}{C_{\theta}} & \frac{C_{\phi}}{C_{\theta}}
\end{array}\right]\left[\begin{array}{l}
p \\
q \\
r
\end{array}\right],
$$

where $T_{(\cdot)}$ denotes $\tan (\cdot)$ and the transformation matrix $Q(\Lambda)$ is bounded according to $\|Q(\Lambda)\|_{F}<Q(\Lambda)_{\max }$ for a known constant $Q(\Lambda)_{\max }$ provided $-(\pi / 2)<\phi<(\pi / 2)$ and $-(\pi / 2)<\theta<(\pi / 2)$ [23]. $M_{B 0}$ is defined as the torque

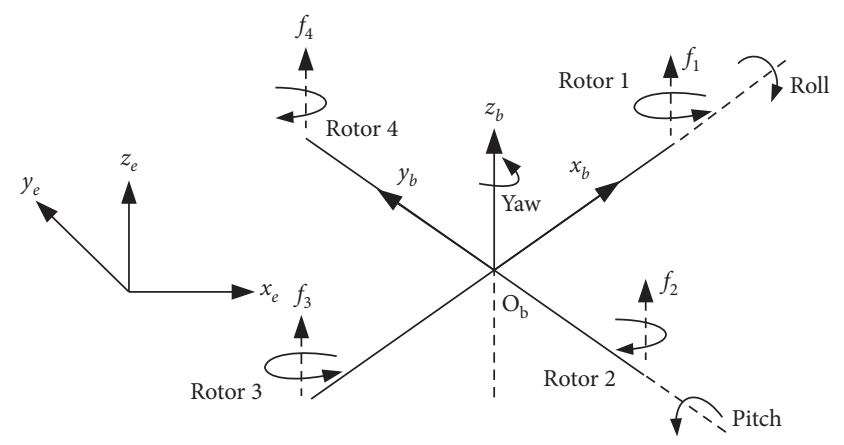

FIgURE 1: Schematic diagram of the quadrotor UAV.

provided by the rotors with respect to the body-fixed frame and is described as follows:

$$
M_{B 0}=\left[\begin{array}{c}
M_{B 0 x} \\
M_{B 0 y} \\
M_{B 0 z}
\end{array}\right]=\left[\begin{array}{c}
l\left(F_{4}-F_{2}\right) \\
l\left(F_{3}-F_{1}\right) \\
\tau_{2}+\tau_{4}-\tau_{1}-\tau_{3}
\end{array}\right],
$$

where $\tau_{i}(i=1, \ldots, 4)=k_{\psi} F_{i}, k_{\psi}$ is a constant, $l$ is the distance between a rotor and the center of mass of the quadrotor, $F_{i}(i=1, \ldots, 4)=k \Omega_{i}^{2}$ denotes the lift provided by the rotor, and we get $\tau_{i}=k_{\psi} k \Omega_{i}^{2}=\tau \Omega_{i}^{2}$, in which $\tau$ represents the antitorque coefficient. Using the Newton-Euler equation, we can get the rotational dynamic equation of the quadrotor:

$$
M_{B 0}=\left(w \times J_{B} w\right)+J_{B} \dot{w}+M_{r}+M_{d},
$$

where $J_{B}=\operatorname{diag}\left(J_{x x}, J_{y y}, J_{z z}\right)$ is a symmetric positive definite constant matrix with $J_{x x}, J_{y y}$, and $J_{z z}$ being the rotary inertia with respect to the $O_{b} x_{b}, O_{b} y_{b}$, and $O_{b} z_{b}$ axes, the signal $\times$ represents the cross multiplication, and $M_{r}$ and $M_{d}$ are the resultant torques due to the gyroscopic effects and the resultant of the aerodynamic frictions torque. They are given as

$$
\begin{aligned}
M_{r} & =\sum_{i=1}^{4} w \times J_{R}\left[0,0,(-1)^{i+1} \Omega_{i}\right]^{T}, \\
M_{d} & =\left[d_{\phi} \dot{\phi}, d_{\theta} \dot{\theta}, d_{\psi} \dot{\psi}\right]^{T},
\end{aligned}
$$

where $J_{R}$ represents the moment of inertia of each rotor and $d_{\phi}, d_{\theta}$, and $d_{\psi}$ are the corresponding aerodynamic drag coefficients.

From (6), the following equation can be obtained:

$$
\dot{w}=\frac{1}{J_{B}}\left[M_{B 0}-M_{r}-M_{d}-w \times\left(J_{B} w\right)\right],
$$

where

$$
w \times J_{B} w=\left[\begin{array}{c}
-r\left(J_{y y} q\right)+q\left(J_{z z} r\right) \\
r\left(J_{x x} p\right)-p\left(J_{z z} r\right) \\
-q\left(J_{x x} p\right)+p\left(J_{z z} q\right)
\end{array}\right] .
$$

With the help of (4), the following equations can be obtained: 


$$
\begin{aligned}
& \phi=\frac{1}{J_{x x}}\left[M_{B 0 x}+\dot{\theta} \dot{\psi}\left(J_{y y}-J_{z z}\right)-J_{R} \dot{\theta} \Omega-d_{\phi} \dot{\phi}\right], \\
& \theta=\frac{1}{J_{y y}}\left[M_{B 0 y}+\dot{\phi} \dot{\psi}\left(J_{z z}-J_{x x}\right)+J_{R} \dot{\phi} \Omega-d_{\theta} \dot{\theta}\right], \\
& \psi=\frac{1}{J_{z z}}\left[M_{B 0 z}+\dot{\theta} \dot{\phi}\left(J_{x x}-J_{y y}\right)-d_{\psi} \dot{\psi}\right],
\end{aligned}
$$

where $\Omega=\Omega_{1}+\Omega_{3}-\Omega_{2}-\Omega_{4}$.

Remark 1. It is worth noting to point out that the roll, pitch, and yaw angles are limited to $(-\pi / 2, \pi / 2)$ which is physically meaningful.

By combing (3) and (10), a nonlinear equation of the quadrotor UAV is given as follows:

$$
\dot{X}=f(X)+g(X) U,
$$

where

$X=\left[x_{1}, x_{2}, x_{3}, x_{4}, x_{5}, x_{6}, x_{7}, x_{8}, x_{9}, x_{10}, x_{11}, x_{12}\right]^{T}=[x, \dot{x}$, $y, \dot{y}, z, \dot{z}, \phi, \dot{\phi}, \theta, \dot{\theta}, \psi, \dot{\psi}]^{T}$ is the state vector and $f(X)$ and $g(X)$ are the smooth functions. The dynamic of quadrotor UAVs can be described as follows:

$$
\left\{\begin{array}{l}
\dot{x}_{1}=x_{2}, \\
\dot{x}_{2}=\left(C_{x_{7}} S_{x_{9}} C_{x_{11}}+S_{x_{7}} S_{x_{11}}\right) U_{1}-a_{1} x_{2}, \\
\dot{x}_{3}=x_{4}, \\
\dot{x}_{4}=\left(C_{x_{7}} S_{x_{9}} S_{x_{11}}-S_{x_{7}} C_{x_{11}}\right) U_{1}-a_{2} x_{4}, \\
\dot{x}_{5}=x_{6} \\
\dot{x}_{6}=\left(C_{x_{7}} C_{x_{9}}\right) U_{1}-g-a_{3} x_{6}, \\
\dot{x}_{7}=x_{8}, \\
\dot{x}_{8}=a_{4} x_{10} x_{12}+a_{5} x_{10} \Omega-a_{6} x_{8}+U_{2}, \\
\dot{x}_{9}=x_{10}, \\
\dot{x}_{10}=a_{7} x_{8} x_{12}+a_{8} x_{8} \Omega-a_{9} x_{10}+U_{3}, \\
\dot{x}_{11}=x_{12}, \\
\dot{x}_{12}=a_{10} x_{8} x_{10}-a_{11} x_{12}+U_{4},
\end{array}\right.
$$

where $g$ is the gravity acceleration and $U_{i}, i=1, \ldots, 4$ are the control inputs which can be expressed as

$$
\left\{\begin{array}{l}
U_{1}=\frac{k\left(\Omega_{1}^{2}+\Omega_{2}^{2}+\Omega_{3}^{2}+\Omega_{4}^{2}\right)}{m} \\
U_{2}=\frac{l k\left(\Omega_{4}^{2}-\Omega_{2}^{2}\right)}{J_{x x}} \\
U_{3}=\frac{l k\left(\Omega_{3}^{2}-\Omega_{1}^{2}\right)}{J_{y y}} \\
U_{4}=\frac{\tau\left(\Omega_{4}^{2}+\Omega_{2}^{2}-\Omega_{1}^{2}-\Omega_{3}^{2}\right)}{J_{z z}}
\end{array}\right.
$$

Dividing the unknown parameters $a_{i}(i=4, \ldots, 11)$ into two parts, known part $a_{i N}$ and unknown part $\Delta a_{i}$, it is expressed as follows:

$$
\begin{aligned}
a_{1} & =\frac{d_{x}}{m}, \\
a_{2} & =\frac{d_{y}}{m}, \\
a_{3} & =\frac{d_{z}}{m}, \\
a_{4 N} & =\frac{J_{y y}-J_{z z}}{J_{x x}}, \\
a_{5 N} & =\frac{J_{R}}{J_{x x}}, \\
a_{6 N} & =\frac{d_{\phi}}{J_{x x}}, \\
a_{7 N} & =\frac{J_{z z}-J_{x x}}{J_{y y}}, \\
a_{9 N} & =\frac{d_{x x}}{J_{y y}}, \\
a_{8 N} & =\frac{J_{R}}{J_{y y}},
\end{aligned}
$$

2.2. Fuzzy Logic Systems (FLSs). The FLS is composed of three main components: fuzzy rule base, fuzzification, and defuzzification operators. The fuzzy rule base comprises a collection of fuzzy "IF-THEN" rules of the following form:

$$
\begin{aligned}
& R^{l}: \text { if } x_{1} \text { is } F_{1}^{l} \text { and } x_{2} \text { is } F_{2}^{l}, \ldots \text { and } x_{n} \text { is } F_{n}^{l} \text {, then } y \text { is } G^{l}, \\
& l=1,2, \ldots, N,
\end{aligned}
$$

where $x=\left[x_{1}, \ldots, x_{n}\right]^{T} \in U$ and $y$ are the FLS input and output, respectively, $N$ is the number of rules, and fuzzy sets $F_{i}^{l}$ and $G^{l}$ are associated with the fuzzy membership functions $\mu_{F^{l}}\left(x_{i}\right)$ and $\mu_{G^{l}}(y)$. Through the singleton function, center average defuzzification, and product inference, the FLS can be expressed as

$$
y(x)=\frac{\sum_{l=1}^{N} \bar{y}_{l} \prod_{i=1}^{n} \mu_{F_{i}^{l}}\left(x_{i}\right)}{\sum_{l=1}^{N}\left[\prod_{i=1}^{n} \mu_{F_{i}^{l}}\left(x_{i}\right)\right]},
$$

where $\bar{y}_{l}=\max _{y \in R} \mu_{G^{l}}(y)$. The fuzzy basis function is defined as 


$$
\xi_{l}=\frac{\prod_{i=1}^{n} \mu_{F_{i}^{l}}\left(x_{i}\right)}{\sum_{l=1}^{N}\left(\prod_{i=1}^{n} \mu_{F_{i}^{l}}\left(x_{i}\right)\right)} .
$$

Denoting $W^{T}=\left[\bar{y}_{1}, \bar{y}_{2}, \ldots, \bar{y}_{N}\right]=\left[W_{1}, W_{2}, \ldots, W_{N}\right]$ and $\xi(x)=\left[\xi_{1}(x), \xi_{2}(x), \ldots, \xi_{N}(x)\right]^{T}$, FLS (15) can be rewritten as

$$
y(x)=W^{T} \xi(x)
$$

Lemma 1. According to [34], FLSs can effectively approximate any continuous nonlinear function with any small approximated error in a compact set. It can be expressed as

$$
\sup _{x \in \Omega}\left|F(x)-W^{T} \xi(x)\right|<\varepsilon,
$$

where the continuous nonlinear function $F(x): \Omega \longrightarrow \mathbb{R}$ with $\Omega \in \mathbb{R}^{n}$ is a compact set, $W^{T} \xi(x)$ is an FLS (17), and $\varepsilon>0$ is the approximated error. Therefore, $F(x)$ can be described as

$$
F(x)=W^{* T} \xi(x)+\sigma^{*}, \quad \forall x \in \Omega \subset \mathbb{R}^{n},
$$

where the minimum approximated error $\left|\sigma^{*}\right| \leq \varepsilon$ and $W^{*}$ is an ideal weight vector and can be defined as

$$
W^{*}=\underset{W \subset \mathbb{R}^{n}}{\arg \min }\left\{\sup _{x \in \Omega}|F(x)-y(x)|<\varepsilon\right\} .
$$

2.3. Performance and the Error Transformation Functions. Similar to [46], the mathematical expression of the prescribed tracking performance is given by

$$
-\kappa_{i} p_{i}(t)<e_{i}(t)<\beta_{i} p_{i}(t)
$$

where $e_{i}(t)=y_{i}-x_{i d}, i=1, \ldots, 6$, are the tracking errors, the performance function $p_{i}(t)$ is defined as a smooth and decreasing positive function, and $\kappa_{i}$ and $\beta_{i}$ are the given positive constants. Moreover, $\kappa_{i} p_{i}(0)$ and $\beta_{i} p_{i}(0)$ represent the lower and upper bounds of the undershoot of $e_{i}(t)$ and $-\kappa_{i} p_{i}(\infty)$ and $\beta_{i} p_{i}(\infty)$ are the maximum allowable size of $e_{i}(t)$.

The error transformation function is chosen as

$$
\Upsilon_{i}\left(S_{i}\right)=\frac{e_{i}(t)}{p_{i}(t)}
$$

where $S_{i}$ is the transformed error variable and $\Upsilon_{i}\left(S_{i}\right)$ is a smooth strictly increasing function with the following properties:

$$
\begin{aligned}
& \lim _{S_{i} \longrightarrow-\infty} \Upsilon_{i}\left(S_{i}\right)=-\kappa_{i}, \\
& S_{i} \stackrel{\lim }{\longrightarrow+\infty} \Upsilon_{i}\left(S_{i}\right)=\beta_{i} .
\end{aligned}
$$

Note that if $S_{i}$ is kept bounded, we have $-\kappa_{i}<\Upsilon_{i}\left(S_{i}\right)<\beta_{i}$, and thus (21) holds. The inverse transformation of $\Upsilon_{i}\left(S_{i}\right)$ can be expressed as

$$
S_{i}=\Upsilon_{i}^{-1}\left(\frac{e_{i}(t)}{p_{i}(t)}\right):=\Theta_{i}\left(\frac{e_{i}(t)}{p_{i}(t)}\right),
$$

where $S_{i}, e_{i}(t)$, and $p_{i}(t)$, are the transformed errors, the output tracking errors, and their corresponding performance functions.

In this paper, we choose

$$
S_{i}=\Theta_{i}\left(\frac{e_{i}(t)}{p_{i}(t)}\right)=\ln \left(\frac{\kappa_{i}+e_{i}(t) / p_{i}(t)}{\beta_{i}-e_{i}(t) / p_{i}(t)}\right),
$$

and differentiating (25) yields

$$
\dot{S}_{i}=\eta_{i} \dot{y}_{i}-\eta_{i} v_{i}
$$

where $\quad \eta_{i}=\left(\partial \Theta / \partial\left(e_{i} / p_{i}\right)\right)\left(1 / p_{i}\right)(i=1, \ldots, 6)$ and $v_{i}=$ $\dot{x}_{i d}+e_{i} \dot{p}_{i} / p_{i}$, where $x_{i d}$ are the reference signals and $e_{i}(t)$ are the output tracking errors. From the properties of the transformation, it is clear that $\eta_{i}$ and $v_{i}$ are bounded and $0<\eta_{i 0} \leq \eta_{i}$.

Remark 2. It can be seen that a new variable $S_{i}$ is introduced through the above transformation process (21)-(25). If the designed control system can guarantee that $S_{i}$ is bounded, then the tracking error $e_{i}$ is bounded and meets formula (21). This means that the tracking error is always kept within the range $\left[-\kappa_{i} p_{i}(t), p_{i}(t)\right]$ or $\left[-p_{i}(t), \beta_{i} p_{i}(t)\right]$. The control objective is to design an adaptive controller so that $S_{i}$ is bounded.

2.4. Nonlinear Observer. For a class of nonlinear systems with $\left(A_{0}, C\right)$ in the observer canonical form is given by

$$
\left\{\begin{array}{l}
\dot{x}=A_{0} x+\sum_{i=1}^{n} b_{i} f_{i}(x)+b_{n} u, \\
y=C^{T} x,
\end{array}\right.
$$

with

$$
\begin{aligned}
A_{0} & =\left[\begin{array}{ccccc}
0 & 1 & 0 & \cdots & 0 \\
0 & 0 & 1 & \cdots & 0 \\
& & \vdots & & \\
0 & 0 & 0 & 1 & 0 \\
0 & 0 & 0 & \cdots & 0
\end{array}\right], \\
C & =\left[\begin{array}{c}
1 \\
0 \\
\vdots \\
0 \\
0
\end{array}\right], \\
b_{i} & =[\underbrace{0, \ldots, 0,1,0, \ldots, 0}_{i-1}]^{T},
\end{aligned}
$$

with $x \in R^{n}, y \in R, u \in R, b_{i} \in R^{n}(i \geq 2)$, and $f(x)$ is the unknown smooth function. The vector $b$ is general and not in a restricted form. Only the output $y$ is assumed to be 
measurable [48]. For the uncertain system (27), the nonlinear state observer is established as

$$
\left\{\begin{array}{l}
\dot{\hat{x}}=A \widehat{x}+\sum_{i=1}^{n} b_{i} \widehat{f}_{i}(\hat{x})+b_{n} u+K y, \\
\hat{y}=C^{T} \widehat{x},
\end{array}\right.
$$

with

$$
\begin{aligned}
A & =\left[\begin{array}{ccccc}
-k_{1} & 1 & 0 & \cdots & 0 \\
-k_{2} & 0 & 1 & \cdots & 0 \\
& & \vdots & & \\
-k_{n-1} & 0 & 0 & 1 & 0 \\
-k_{n} & 0 & 0 & \cdots & 0
\end{array}\right], \\
K & =\left[\begin{array}{c}
-k_{1} \\
-k_{2} \\
\vdots \\
-k_{n-1} \\
-k_{n}
\end{array}\right],
\end{aligned}
$$

where $\hat{x}$ is the estimation of the state $x$ and $K$ is the observer gain vector; $K$ is chosen so that the characteristic polynomial of $A-K C^{T}$ is strictly Hurwitz. Thus, for a given a matrix $Q_{1}=Q_{1}^{T}>0$, there exists a positive definite matrix $P$ such that

$$
A^{T} P+P A=-Q_{1} .
$$

The function $\widehat{f}(\widehat{x})$ is the estimation of $f(x)$. In the next section, we choose FLSs to approximate $f(x)$. According to (27) and (29), the observer error can be expressed as

$$
\dot{\tilde{x}}=A \tilde{x}+\sum_{i=1}^{n} b_{i} \tilde{W}_{i}^{T} \xi\left(\widehat{x}_{i}\right)+\sum_{i=1}^{n} b_{i} \varepsilon_{i}=A \tilde{x}+\sum_{i=1}^{n} b_{i} \tilde{W}_{i}^{T} \xi\left(\widehat{x}_{i}\right)+\bar{\varepsilon},
$$

where $\tilde{x}=x-\widehat{x}, \widetilde{W}_{i}=W_{i}^{*}-\widehat{W}_{i}$, and $\bar{\varepsilon}=\left[\varepsilon_{1}, \ldots, \varepsilon_{n}\right]^{T}$.

\section{The Process of the Controller Design}

In this section, an adaptive FLS dynamic surface slidingmode control scheme is proposed for position and attitude trajectory tracking control. The structure of the proposed control scheme is shown in Figure 2. The recursive design procedure contains two parts. Part 1 is the position trajectory tracking control and part 2 is the attitude trajectory tracking control. Each part contains three design steps, which are shown in Tables 1 and 2. The details of the controller design process are shown in Appendix A.

In Table $1, S_{i},(i=1, \ldots, 6)$ are the surface errors and $\bar{x}_{j d},(j=2,4,6)$ are the virtual control signals in Step 1, Step 3, and Step 5, respectively; (T1.3), (T1.9), and (T1.15) represent the virtual control signal pass through a first-order filter to obtain a new variable $x_{j d},(j=2,4,6)$ with the time constant $\tau_{j},(j=2,4,6)$; (T1.6), (T1.12), and (T1.18) are the adaptive laws, and $c_{i},(i=1, \ldots, 6), \lambda_{j}, \mu_{j},(j=1,2,3)$ are the design positive parameters. It should be noted that $\chi_{i},(i=1,2,3)$ are the virtual control given by
$\chi_{1}=\left(C_{x_{7}} S_{x_{9}} C_{x_{11}}+S_{x_{7}} S_{x_{11}}\right) U_{1}, \chi_{2}=\left(C_{x_{7}} S_{x_{9}} S_{x_{11}}-S_{x_{7}} C_{x_{11}}\right) U_{1}$, and $\chi_{3}=\left(C_{x_{7}} C_{x_{9}}\right) U_{1}$.

In Table $2, S_{i},(i=7, \ldots, 12)$ are the surface errors and $\bar{x}_{j d},(j=8,10,12)$ are the virtual control signals in Step 7, Step 9, and Step 11, respectively; (T2.4), (T2.11), and (T2.18) represent the virtual control signal pass through a first-order filter to obtain a new variable $x_{j d},(j=8,10,12)$ with the time constant $\tau_{j},(j=8,10,12)$; (T2.7), (T2.14), and (T2.21) are the adaptive laws, and $c_{i},(i=7, \ldots, 12), \lambda_{j}, \mu_{j},(j=4,5,6)$ are the design positive parameters; $k_{1}$ and $k_{2}$ are the observer gain.

It should be note that $\widehat{\theta}_{i},(i=1, \ldots, 6)$ are the estimations of $\theta_{i}$ with $\theta_{i}=\left\|W_{i}^{*}\right\|^{2}$, and $\left\|W_{i}^{*}\right\|^{2}$ and $\xi_{i}\left(X_{i}\right)$ are the ideal weight vector and fuzzy basis function vector of FLSs which are used to approximate the unknown continuous nonlinear function at each design step.

Remark 3. For the attitude trajectory tracking control sysytem, $\chi_{1}, \chi_{2}$, and $\chi_{3}$ can be regarded as known and the input $U_{1}$ can be solved. The denominator of $U_{1}$ will not cause singularity since the yaw angle is limited to $(-\pi / 2, \pi / 2)$.

Remark 4. In the traditional sliding-mode control method, the existence of the signum function will cause chattering in the control system. In practical applications, the saturation function sat(.) [49] or the hyperbolic tangent function $\tanh (\cdot)$ [50] are generally used to eliminate the chatting phenomenon.

\section{Stability and Prescribed Tracking Performance Analysis}

First of all, define the filter error

$$
y_{i}=x_{i d}-\bar{x}_{i d}, \quad i=2,4,6,7,8,9,10,12,
$$

from (A.3), (A.13), (A.23), and (A.37). We have

$$
\dot{x}_{i d}=-\frac{y_{i}}{\tau_{i}}, \quad i=2,4,6,7,8,9,10,12 .
$$

Differentiating the boundary errors yields

$$
\dot{y}_{2}=\dot{x}_{2 d}-\dot{\bar{x}}_{2 d}=-\frac{y_{2}}{\tau_{2}}+k_{1}\left(\dot{x}_{1}-\dot{\hat{x}}_{1}\right)-\dot{v}_{1}+\frac{\dot{S}_{1} c_{1}}{\eta_{1}}-\frac{S_{1} c_{1}}{\eta_{1}^{2}},
$$

from which we have

$$
\dot{y}_{2}=-\frac{y_{2}}{\tau_{2}}+B_{2}\left(S_{1}, S_{2}, y_{2}, \ddot{x}_{1 d}\right) \text {. }
$$

By the same token, one has

$$
\dot{y}_{i}=-\frac{y_{i}}{\tau_{i}}+B_{i}(\cdot)
$$

where $B_{i}(\cdot), i=2,4,6,7,8,9,10,12$, are the continuous functions. From (37), the following inequality holds:

$$
y_{i} \dot{y}_{i} \leq-\frac{y_{i}^{2}}{\tau_{i}}+B_{i}\left|y_{i}\right| \text {. }
$$




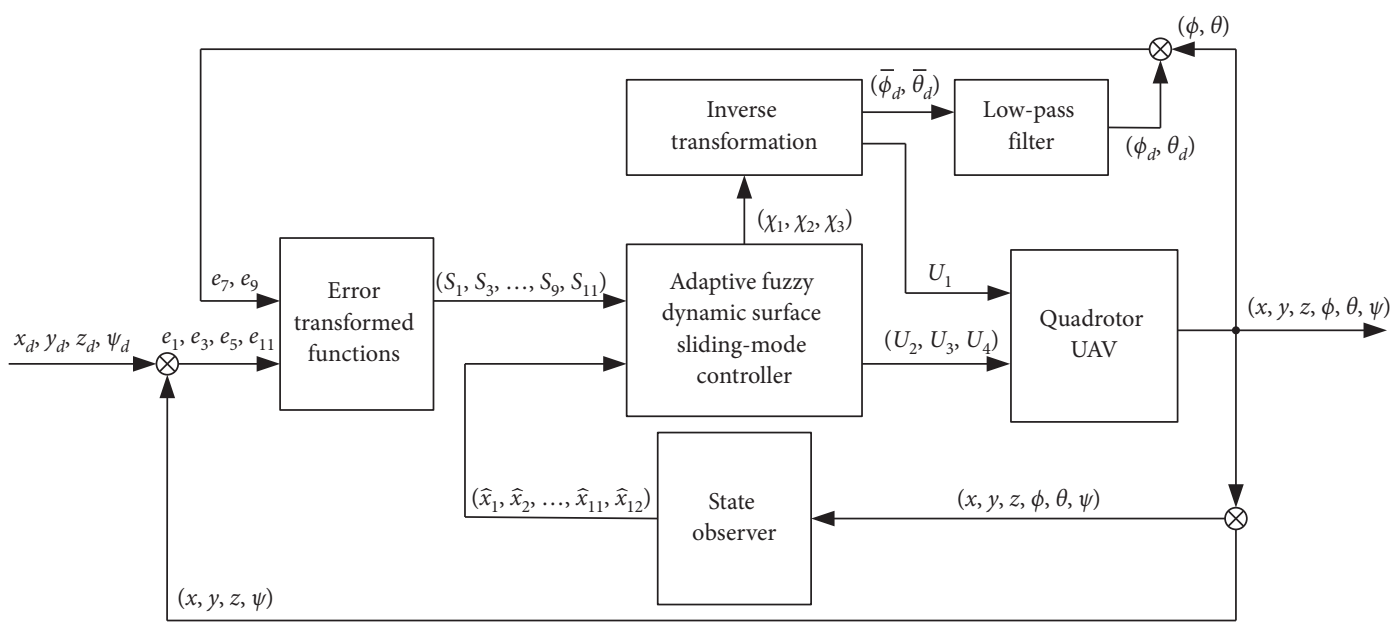

FIGURE 2: Structure of the proposed control scheme.

TABLE 1: The proposed DSCSM design for position trajectory control.

Step 1

$S_{1}=\Theta_{1}\left(\left(e_{1}(t)\right) /\left(p_{1}(t)\right)\right)$,

$\dot{\hat{x}}_{1}=\hat{x}_{2}+k_{1}\left(x_{1}-\hat{x}_{1}\right), \dot{\hat{x}}_{2}=\hat{f}\left(\hat{x}_{2}\right)+\chi_{1}+k_{2}\left(x_{1}-\hat{x}_{1}\right)$,

$\bar{x}_{2 d}=-k_{1}\left(x_{1}-\hat{x}_{1}\right)+v_{1}-S_{1} c_{1} / \eta_{1}, \tau_{2} \dot{x}_{2 d}+x_{2 d}=\bar{x}_{2 d}, x_{2 d}(0)=\bar{x}_{2 d}(0)$.

Step 2

$S_{2}=\widehat{x}_{2}-x_{2 d}$,

$\chi_{1}=-c_{2} S_{2}-(1 / 2) S_{2} \widehat{\theta}_{1} \xi_{1}^{T}\left(X_{1}\right) \xi_{1}\left(X_{1}\right)-k_{2}\left(x_{1}-\hat{x}_{1}\right)+\dot{x}_{2 d}-\mu_{1} \operatorname{sgn}\left(S_{2}\right)$,

$\hat{\theta}_{1}=(1 / 2) S_{2}^{2} \xi_{1}^{T}\left(X_{1}\right) \xi_{1}\left(X_{1}\right)-\lambda_{1} \widehat{\theta}_{1}, \hat{\theta}_{1}(0) \geq 0$

Step 3

$S_{3}=\Theta_{3}\left(\left(e_{3}(t)\right) /\left(p_{1}(t)\right)\right)$,

$\dot{\hat{x}}_{3}=\hat{x}_{4}+k_{1}\left(x_{3}-\hat{x}_{3}\right), \dot{\hat{x}}_{4}=\widehat{f}\left(\hat{x}_{4}\right)+\chi_{2}+k_{2}\left(x_{3}-\hat{x}_{3}\right)$,

$\bar{x}_{4 d}=-k_{1}\left(x_{3}-\hat{x}_{3}\right)+v_{2}-S_{3} c_{3} / \eta_{2}, \tau_{4} \dot{x}_{4 d}+x_{4 d}=\bar{x}_{4 d}, x_{4 d}(0)=\bar{x}_{4 d}(0)$.

Step 4

$S_{4}=\hat{x}_{4}-x_{4 d}$,

$\chi_{2}=-c_{4} S_{4}-(1 / 2) S_{4} \widehat{\theta}_{2} \xi_{2}^{T}\left(X_{2}\right) \xi_{2}\left(X_{2}\right)-k_{2}\left(x_{3}-\widehat{x}_{3}\right)+\dot{x}_{4 d}-\mu_{2} \operatorname{sgn}\left(S_{4}\right)$,

$\theta_{2}=(1 / 2) S_{4}^{2} \xi_{2}^{T}\left(X_{2}\right) \xi_{2}\left(X_{2}\right)-\lambda_{2} \hat{\theta}_{2}, \theta_{2}(0) \geq 0$

Step 5

$S_{5}=\Theta_{5}\left(\left(e_{5}(t)\right) /\left(p_{1}(t)\right)\right)$,

$\dot{\hat{x}}_{5}=\hat{x}_{6}+k_{1}\left(x_{5}-\hat{x}_{5}\right), \dot{\hat{x}}_{6}=\hat{f}\left(\hat{x}_{6}\right)+\chi_{3}+k_{2}\left(x_{5}-\hat{x}_{5}\right)$,

$\bar{x}_{6 d}=-k_{1}\left(x_{5}-\hat{x}_{5}\right)+v_{3}-S_{5} c_{5} / \eta_{3}, \tau_{6} \dot{x}_{6 d}+x_{6 d}=\bar{x}_{6 d}, x_{6 d}(0)=\bar{x}_{6 d}(0)$.

Step 6

$S_{6}=\hat{x}_{6}-x_{6 d}$,

$\chi_{3}=-c_{6} S_{6}-(1 / 2) S_{6} \widehat{\theta}_{3} \xi_{3}^{T}\left(X_{3}\right) \xi_{3}\left(X_{3}\right)-k_{2}\left(x_{5}-\widehat{x}_{5}\right)+\dot{x}_{6 d}-\mu_{3} \operatorname{sgn}\left(S_{6}\right)$,

$\hat{\theta}_{3}=(1 / 2) S_{6}^{2} \xi_{3}^{T}\left(X_{3}\right) \xi_{3}\left(X_{3}\right)-\lambda_{3} \widehat{\theta}_{3}, \widehat{\theta}_{3}(0) \geq 0$.

Consider the Lyapunov Function candidate as

$$
\Gamma=\frac{1}{2} \tilde{x}^{T} P \widetilde{x}+\sum_{i=1}^{6} \Gamma_{i}+\sum_{i=1}^{6} \frac{1}{2} y_{2 i}^{2}+\frac{1}{2} y_{7}^{2}+\frac{1}{2} y_{9}^{2},
$$

where $\tilde{x}=\left[\tilde{x}_{1}, \tilde{x}_{2}, \ldots, \tilde{x}_{12}\right]^{T}$ and $\Gamma_{i}$ are defined in (A.6)-(A.45).

Theorem 1. For the quadrotor system (12) and the state observer (29), the adaptive laws (T1.6), (T1.12), (T1.18), (T2.7), (T2.14), and (T2.21) and the control input (T2.6), (T2.13), and (T2.20) are given in Table 1 and Table 2; if $\Gamma(0)$ satisfies $\Gamma(0) \leq P,(P \geq 0)$, then, by properly selecting the design parameters $c_{i},(i=1, \ldots, 12), \lambda_{j}, \mu_{j},(j=1, \ldots, 6)$ and the time constant of the low-pass filter $\tau_{2}, \tau_{4}, \tau_{6}, \tau_{7}, \tau_{8}, \tau_{9}, \tau_{10}$, and $\tau_{12}$ appropriately, all the signals in the closed-loop system are semiglobal uniformly bounded. In addition, the tracking error of position and attitude angle can converge to an arbitrarily residual set and is always kept in the prespecified cures.

Proof. Please see Appendix B for details.

\section{Hardware in the Loop Simulation Results}

In this paper, the hardware-in-loop testing platform is used to verify the effectiveness of the proposed control scheme. The experiment environment and the experimental system architecture are shown in Figures 3 and 4, where the following components are included: 
TABLE 2: The proposed DSCSM design for attitude trajectory control.

Step 7

$S_{7}=\Theta_{7}\left(\left(e_{7}(t)\right) /\left(p_{\psi}(t)\right)\right)$,

$\dot{\hat{x}}_{7}=\hat{x}_{8}+k_{1}\left(x_{7}-\hat{x}_{7}\right)$,

$\dot{\hat{x}}_{8}=a_{4 N} \widehat{x}_{10} \widehat{x}_{12}+a_{5 N} \widehat{x}_{10} \Omega-a_{6 N} \widehat{x}_{8}+\hat{f}\left(\widehat{x}_{8}, \widehat{x}_{10}, \widehat{x}_{12}\right)+U_{2}+k_{2}\left(x_{7}-\hat{x}_{7}\right)$,

$\bar{x}_{8 d}=-k_{1}\left(x_{7}-\hat{x}_{7}\right)+v_{4}-S_{7} c_{7} / \eta_{4}, \tau_{8} \dot{x}_{8 d}+x_{8 d}=\bar{x}_{8 d}, x_{8 d}(0)=\bar{x}_{8 d}(0)$.

Step 8

$S_{8}=\hat{x}_{8}-x_{8 d}$,

$U_{2}=-c_{8} S_{8}-(1 / 2) S_{8} \widehat{\theta}_{4} \xi_{4}^{T}\left(X_{4}\right) \xi_{4}\left(X_{4}\right)-k_{2}\left(x_{7}-\widehat{x}_{7}\right)-a_{4} N \widehat{x}_{10} \widehat{x}_{12}-a_{5 N} \widehat{x}_{10} \Omega+a_{6 N} \widehat{x}_{8}+\dot{x}_{8 d}-\mu_{4} \operatorname{sgn}\left(S_{8}\right)$,

$\widehat{\theta}_{4}=(1 / 2) S_{8}^{2} \xi_{4}^{T}\left(X_{4}\right) \xi_{4}\left(X_{4}\right)-\lambda_{4} \hat{\theta}_{4}, \hat{\theta}_{4}(0) \geq 0$

Step 9

$S_{9}=\Theta_{9}\left(\left(e_{9}(t)\right) /\left(p_{\psi}(t)\right)\right)$,

$\dot{\hat{x}}_{9}=\hat{x}_{10}+k_{1}\left(x_{9}-\widehat{x}_{9}\right)$,

$\hat{\dot{x}}_{10}=a_{7 N} \widehat{x}_{8} \widehat{x}_{12}+a_{8 N} \widehat{x}_{8} \Omega-a_{9 N} \widehat{x}_{10}+\hat{f}\left(\widehat{x}_{8}, \hat{x}_{10}, \widehat{x}_{12}\right)+U_{3}+k_{2}\left(x_{9}-\hat{x}_{9}\right)$,

$\bar{x}_{10 d}=-k_{1}\left(x_{9}-\hat{x}_{9}\right)+v_{5}-S_{9} c_{9} / \eta_{5}, \tau_{10} \dot{x}_{10 d}+x_{10 d}=\bar{x}_{10 d}, x_{10 d}(0)=\bar{x}_{10 d}(0)$.

Step 10

$S_{10}=\widehat{x}_{10}-x_{10 d}$

$U_{3}=-c_{10} S_{10}-(1 / 2) S_{10} \hat{\theta}_{5} \xi_{5}^{T}\left(X_{5}\right) \xi_{5}\left(X_{5}\right)-k_{2}\left(x_{9}-\hat{x}_{9}\right)-a_{7 N} \widehat{x}_{8} \hat{x}_{12}-a_{8 N} \widehat{x}_{8} \Omega+a_{9 N} \hat{x}_{10}+\dot{x}_{10 d}-\mu_{5} \operatorname{sgn}\left(S_{10}\right)$,

$\widehat{\theta}_{5}=(1 / 2) S_{10}^{2} \xi_{5}^{T}\left(X_{5}\right) \xi_{5}\left(X_{5}\right)-\lambda_{5} \hat{\theta}_{5}, \widehat{\theta}_{5}(0) \geq 0$.

Step 11

$S_{11}=\Theta_{11}\left(\left(e_{11}(t)\right) /\left(p_{\psi}(t)\right)\right)$,

$\dot{\hat{x}}_{11}=\hat{x}_{12}+k_{1}\left(x_{11}-\widehat{x}_{11}\right)$

$\dot{\hat{x}}_{12}=a_{10 N} \widehat{x}_{8} \widehat{x}_{10}-a_{11 N} \widehat{x}_{12}+\hat{f}\left(\widehat{x}_{8}, \hat{x}_{10}, \widehat{x}_{12}\right)+U_{4}+k_{2}\left(x_{11}-\widehat{x}_{11}\right)$,

$\bar{x}_{12 d}=-k_{1}\left(x_{11}-\hat{x}_{11}\right)+v_{6}-S_{11} c_{11} / \eta_{6}, \tau_{12} \dot{x}_{12 d}+x_{12 d}=\bar{x}_{12 d}, x_{12 d}(0)=\bar{x}_{12 d}(0)$.

Step 12

$S_{12}=\widehat{x}_{12}-x_{12 d}$

$U_{4}=-c_{12} S_{12}-(1 / 2) S_{12} \widehat{\theta}_{6} \xi_{6}^{T}\left(X_{6}\right) \xi_{6}\left(X_{6}\right)-k_{2}\left(x_{11}-\widehat{x}_{11}\right)-a_{10 N} \widehat{x}_{8} \hat{x}_{10}+a_{11 N} \hat{x}_{12}+\dot{x}_{12 d}-\mu_{6} \operatorname{sgn}\left(S_{12}\right)$,

$\widehat{\theta}_{6}=(1 / 2) S_{12}^{2} \xi_{6}^{T}\left(X_{6}\right) \xi_{6}\left(X_{6}\right)-\lambda_{6} \hat{\theta}_{6}, \widehat{\theta}_{6}(0) \geq 0$.

(i) NI PXIe-1082, the MT real-time simulator (RTS) with Kintex-7 325T FPGA chip and 16bits synchronized analog $\mathrm{I} / \mathrm{O}$ with a data transfer rate of $1 \mathrm{MS} / \mathrm{s}$. The simulator supports FPGA simulation for the quadrotor UAV system. The device accepts the control signal and calculates the response of the system in real time and outputs to the controller box.

(ii) NI PXIe-1071, the MT Rapid Control Prototype (RCP), with Kintex-7 325T FPGA @Xilinx and 16 analog I/O channels with a transmission rate of $1 \mathrm{MS} / \mathrm{s}$. This device is used to realize the real-time running of the control code and send the control signals to the quadrotor UAV simulation model which is running on the MT real-time simulator.

(iii) Adapter plate: it is used to realize the signal connection between the RTS and RCP. The RTS, RCP, and the signal adapter board comprise a closed loop experimental system.

(iv) Host computer: uses the StarSim RCP software to download the Matlab/simulink quadrotor UAV system model and the control algorithm into the RTS and RCP, respectively.

In this section, the effectiveness and the performance of the proposed adaptive dynamic surface sliding-mode output feedback control method are showed by the following experiments. Different scenarios are considered, including normal case and model uncertainty cases to demonstrate the

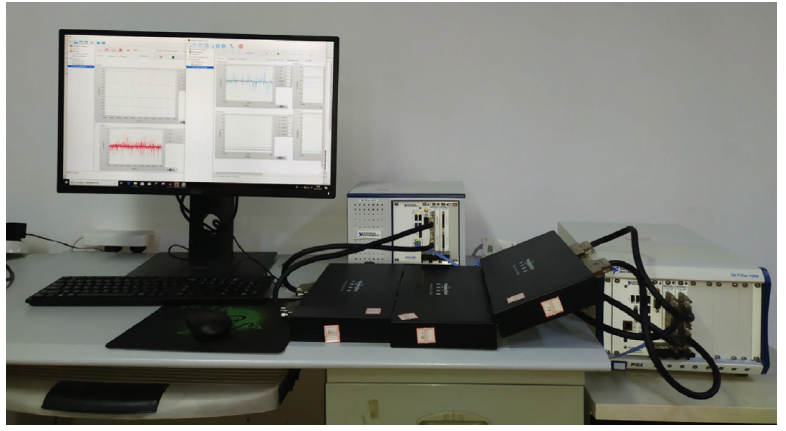

FIgUre 3: Actual experimental environment.

robustness of the proposed controllerlenleadertwodots The parameters of the quadrotor UAV adopted in this study are described in Table 3 [17]. In the experiment, the desired trajectory of the position and yaw angle desired trajectory $\left\{x_{d}(t), y_{d}(t), z_{d}(t), \psi_{d}(t)\right\}$ is chosen as $\{\cos (t), \sin (t), 0.5(t), \sin (0.5 t)\}$. The performance functions are selected as $p_{1}(t)=\left(p_{10}-p_{1 \infty}\right) e^{-l t}+p_{1 \infty}$, with the parameters $\quad p_{10}=1.5, p_{100}=0.055, l=1$, and $\kappa_{1}=\beta_{1}=1$. $p_{\psi}(t)=0.6 * e^{-t}+0.04$. The controller parameters chosen for simulation are $c_{1}=c_{2}=c_{3}=c_{4}=0.6, c_{5}=c_{6}=0.55$, $c_{7}=c_{8}=c_{9}=c_{10}=0.6, c_{11}=c_{12}=0.6, \lambda_{1}=\lambda_{2}=\lambda_{3}=\lambda_{4}=$ $\lambda_{5}=\lambda_{6}=0.02$, and $\tau_{i}=0.005,(i=2,4,6,7,8,9,10,12)$. To demonstrate the effectiveness of the proposed controller, the following different cases are considered and comparisons are conducted: 


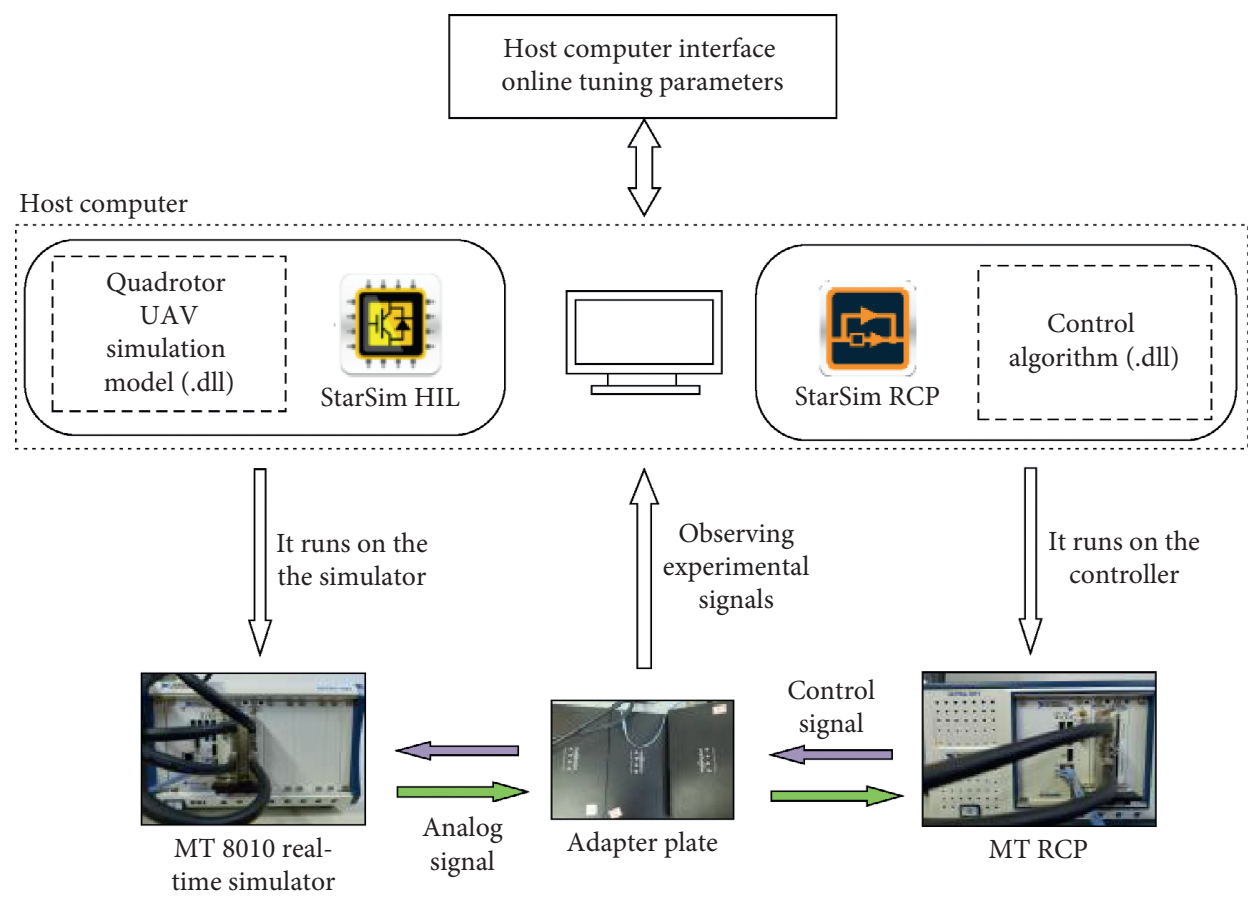

Figure 4: The experimental system architecture.

TABle 3: Quadrotor parameters.

\begin{tabular}{lccccc}
\hline Symbol & Case 1 & Case 2 & Case 3 & Case 4 & Units \\
\hline$m$ & 1.4 & 1.4 & 1.4 & 1.4 & $\mathrm{~kg}$ \\
$k$ & 2.98 & 2.98 & 2.98 & 2.98 & $10^{-6} \mathrm{~N} \cdot \mathrm{s}^{2} \cdot \mathrm{rad}^{-2}$ \\
$l$ & 0.2 & 0.2 & 0.2 & 0.2 & $\mathrm{~m}$ \\
$\tau$ & 1.14 & 1.14 & 1.14 & 1.14 & $10^{-7} \mathrm{~N} \cdot \mathrm{s}^{2} \cdot \mathrm{rad}^{-2}$ \\
$d_{\phi}, d_{\theta}, d_{\psi}$ & 1.2 & 1.2 & 1.2 & 1.2 & $10^{-2} \mathrm{~N} \cdot \mathrm{s} \cdot \mathrm{rad}^{-1}$ \\
$J_{R}$ & 5 & 5 & 5 & 5 & $10^{-5} \mathrm{~N} \cdot \mathrm{s}^{2} \cdot \mathrm{rad}^{-1}$ \\
$J_{x x}, J_{y y}$ & 0.03 & 0.03 & 0.03 & 0.03 & $\mathrm{~N} \cdot \mathrm{s}^{2} \cdot \mathrm{rad}^{-1}$ \\
$J_{z z}$ & 0.04 & 0.046 & 0.052 & 0.06 & $\mathrm{~N} \cdot \mathrm{s}^{2} \cdot \mathrm{rad}^{-1}$ \\
\hline
\end{tabular}

Case 1: normal case: we assume that there are no uncertainties in the model, and all parameters of the quadrotor are normal. The initial state vector is set to be $x(0)=[0.02,0,0.02,0,0,0,0,0,0,0,0.1,0]^{T}$. And, the fuzzy membership function is adopted as $\mu_{F_{k}^{l}}=\exp \left(-\left(\left(\hat{x}_{k}-6+2 l\right)^{2}\right) / 2\right)$, where $l=1, \ldots, 5$ and $k \stackrel{k}{=} 2,6,8,10,12$.

Cases 2, 3, and 4: uncertainty (15\%,30\%, and 50\% added) in rotary inertia: in these cases, we consider three different model uncertainties $15 \%, 30 \%$, and $50 \%$ separately added in the yaw axis.

The experimental results are shown in Figures 5-15. Figures 5-9 illustrate the comparison experimental results of the tracking trajectory in Casel between the proposed control method and the traditional PID control method. From Figures 6-9, it can be seen clearly that all the tracking errors of the position and yaw angles of the proposed control scheme are always kept within the performance function curves. That is, the control method proposed in this paper obtains much better control performance by comparing with the traditional PID control scheme. Figure 10 shows the

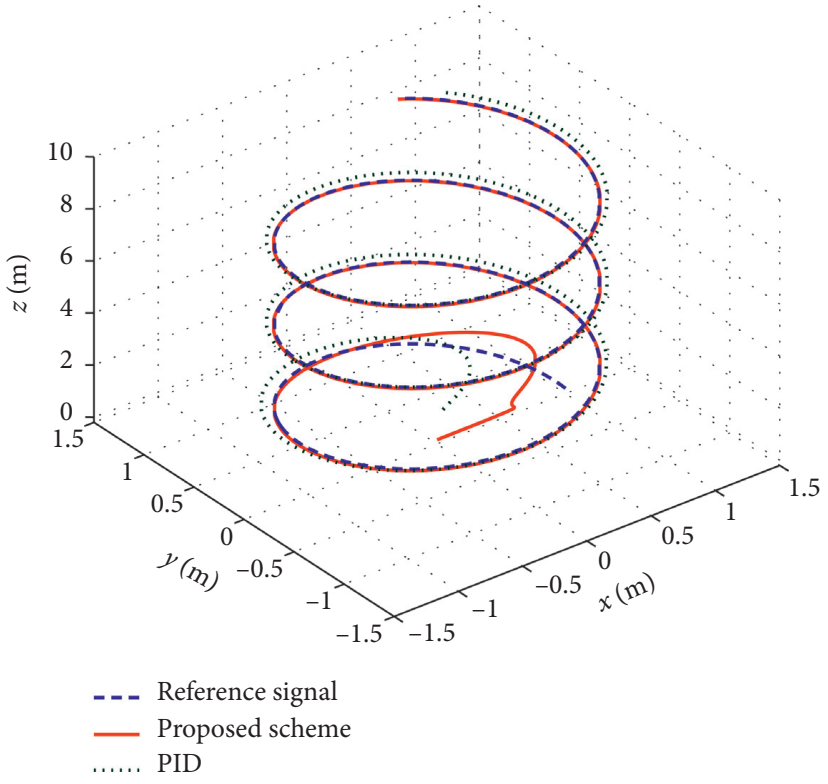

FIGURE 5: Space diagram of position in the normal case.

control signals. Figure 11 shows the response curves of roll and pitch angles. Figures 12 and 13 show the change of six adaptive parameters. Figures 14 and 15 show the trajectories of $x_{i}$ and $\widehat{x}_{i}, \quad(i=2,4,6,8,10,12)$. We can see that the proposed state observer can quickly approximate the output of the system. Figure 16 shows the 3D tracking trajectory with uncertainties $15 \%, 30 \%$, and $50 \%$ added in the yaw rotating axes. Figure 17 illustrates the results of the tracking error under cases 2,3, and 4. Also, the maximum value of the tracking error (MVTE) and the root mean square value of the tracking error (RMSVTE) in the steady state $(t>5 \mathrm{~s})$ are 


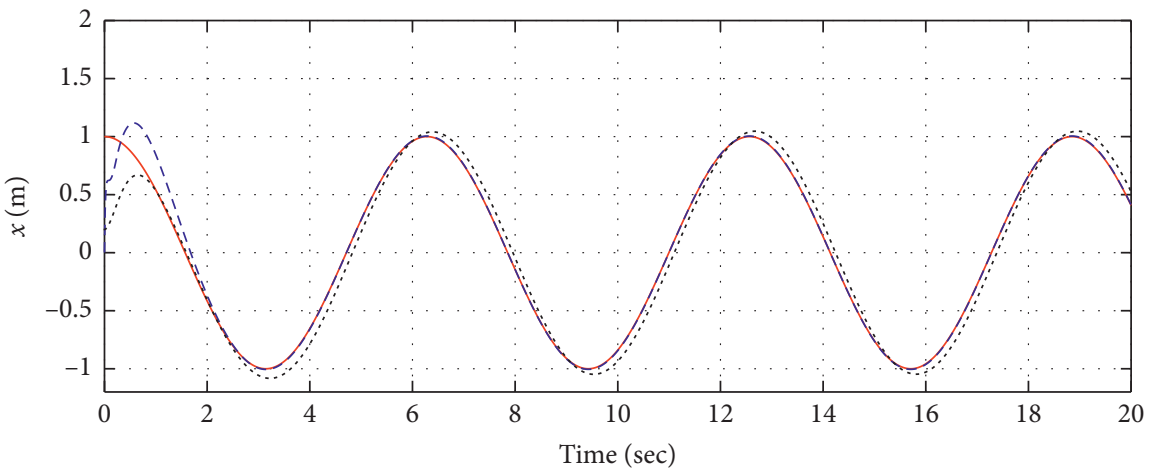

$-x_{d}$

- - Proposed scheme

..... PID

(a)

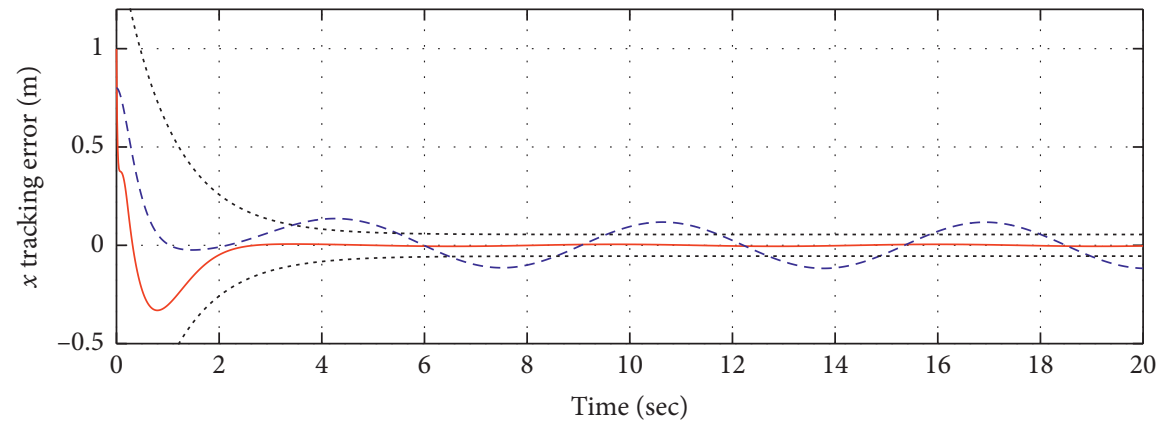

_ Proposed scheme

- - - PID

$\cdots+/-p_{1}(t)$

(b)

FIGURE 6: The tracking trajectory of $x_{d}, x$, and the tracking error.

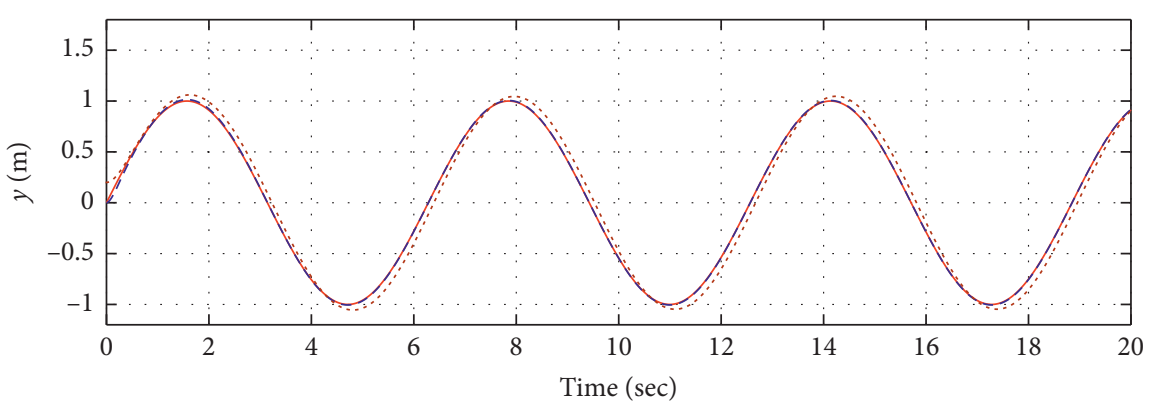

$-y_{d}$

- - - Proposed scheme

..... PID

(a)

Figure 7: Continued. 


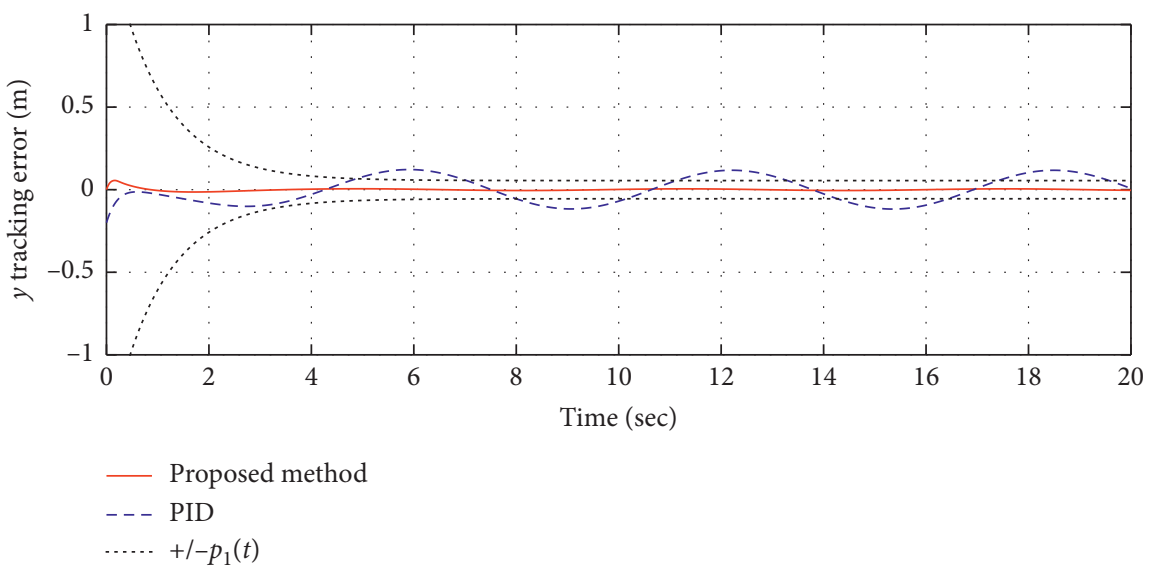

(b)

Figure 7: The tracking trajectory of $y_{d}, y$, and the tracking error.

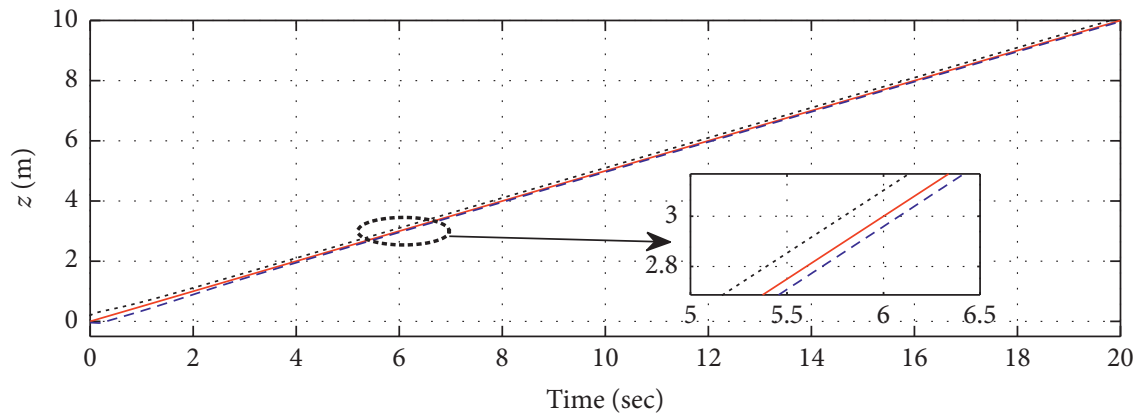

$-z_{d}$

- - - Proposed scheme

….. PID

(a)

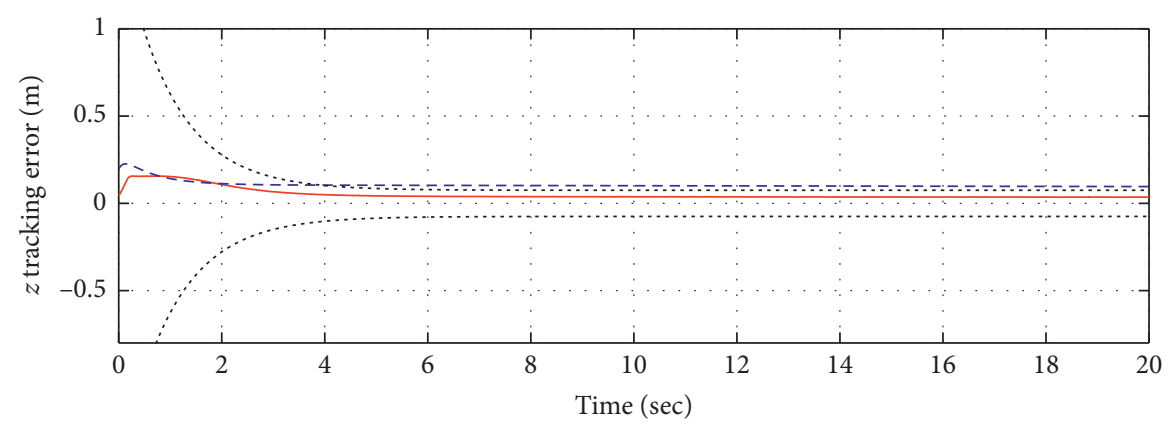

— Proposed scheme

- - - PID

$\cdots++/-p_{1}(t)$

(b)

FIGURE 8: The tracking trajectory of $z_{d}, z$, and the tracking error. 


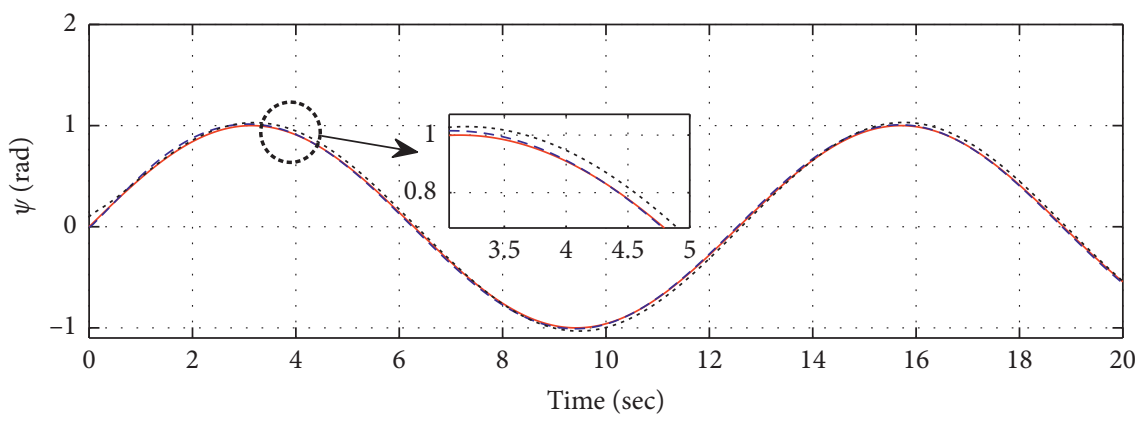

$-\psi_{d}$

- - - Proposed scheme

...... PID

(a)

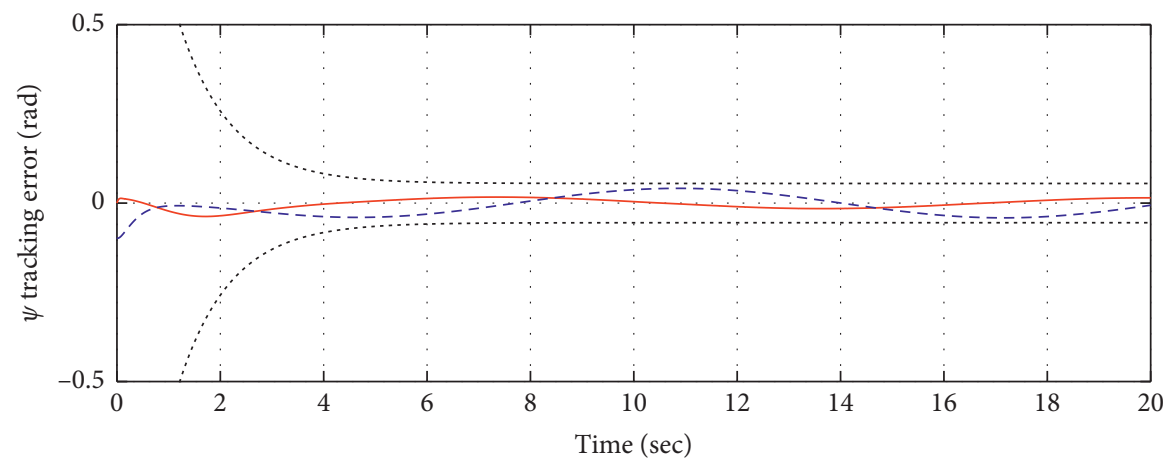

Proposed scheme

- - - PID

$\cdots+/-p_{\psi}(t)$

(b)

FIGURE 9: The tracking trajectory of $\psi_{d}, \psi$, and the tracking error.
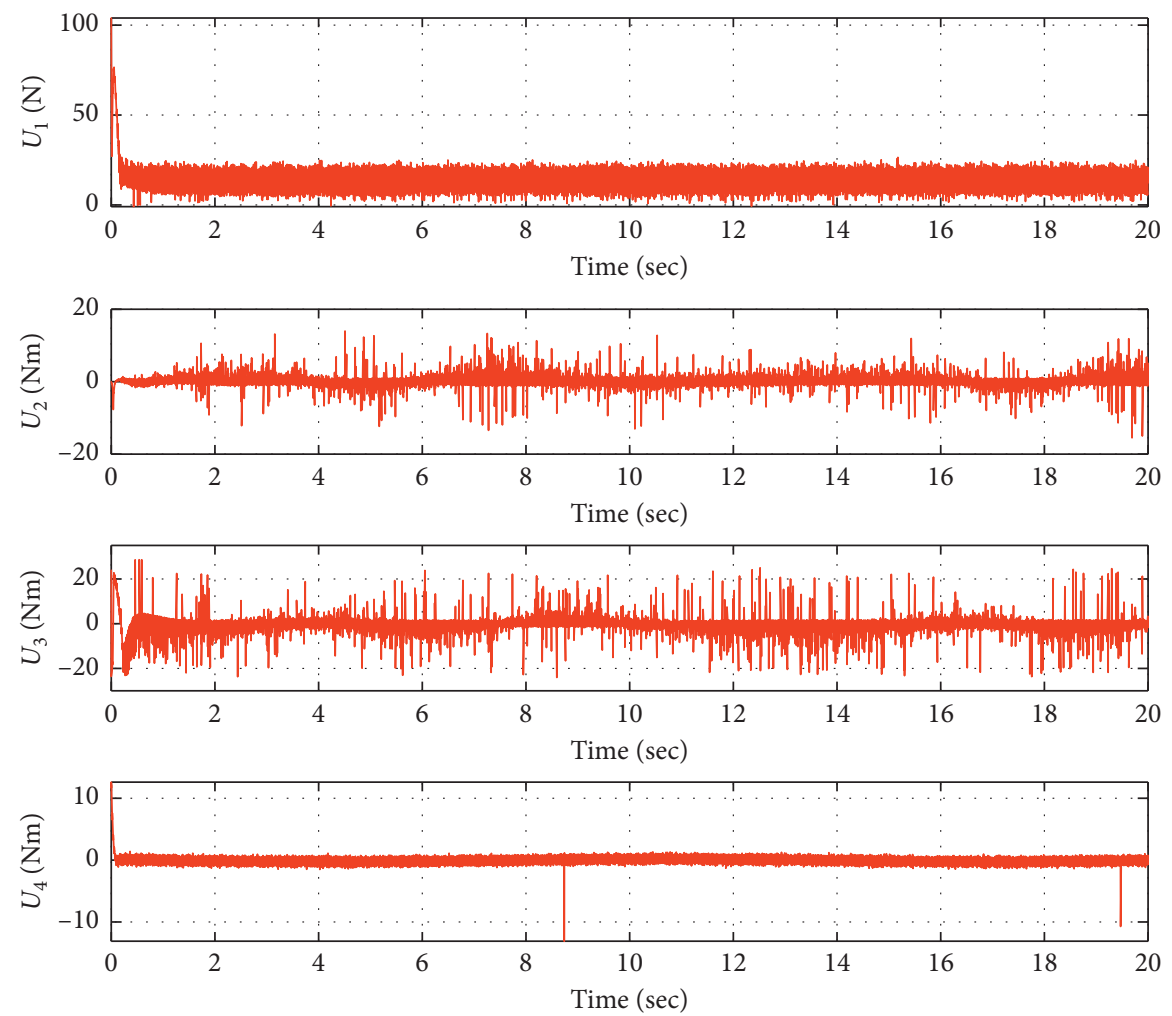

Figure 10: Control signals. 


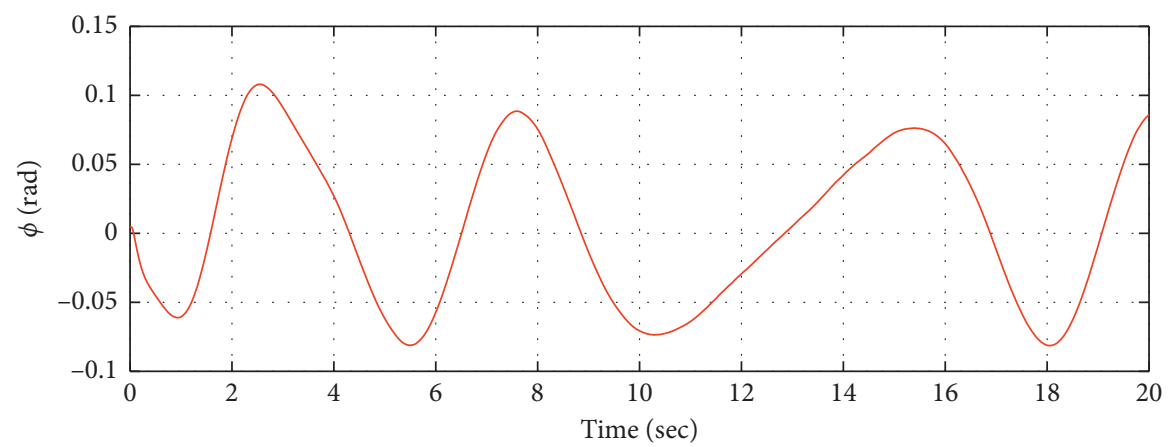

(a)

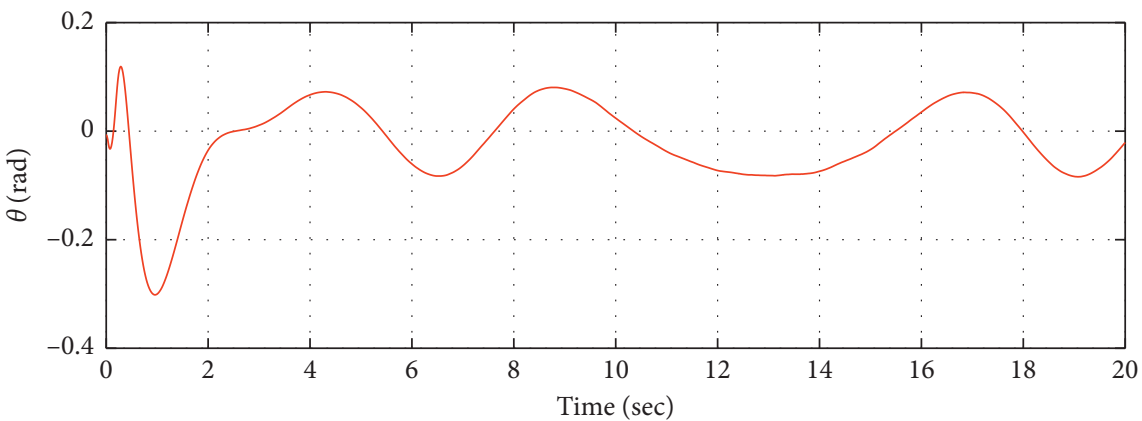

(b)

FIGURE 11: Change of roll and pitch angles.
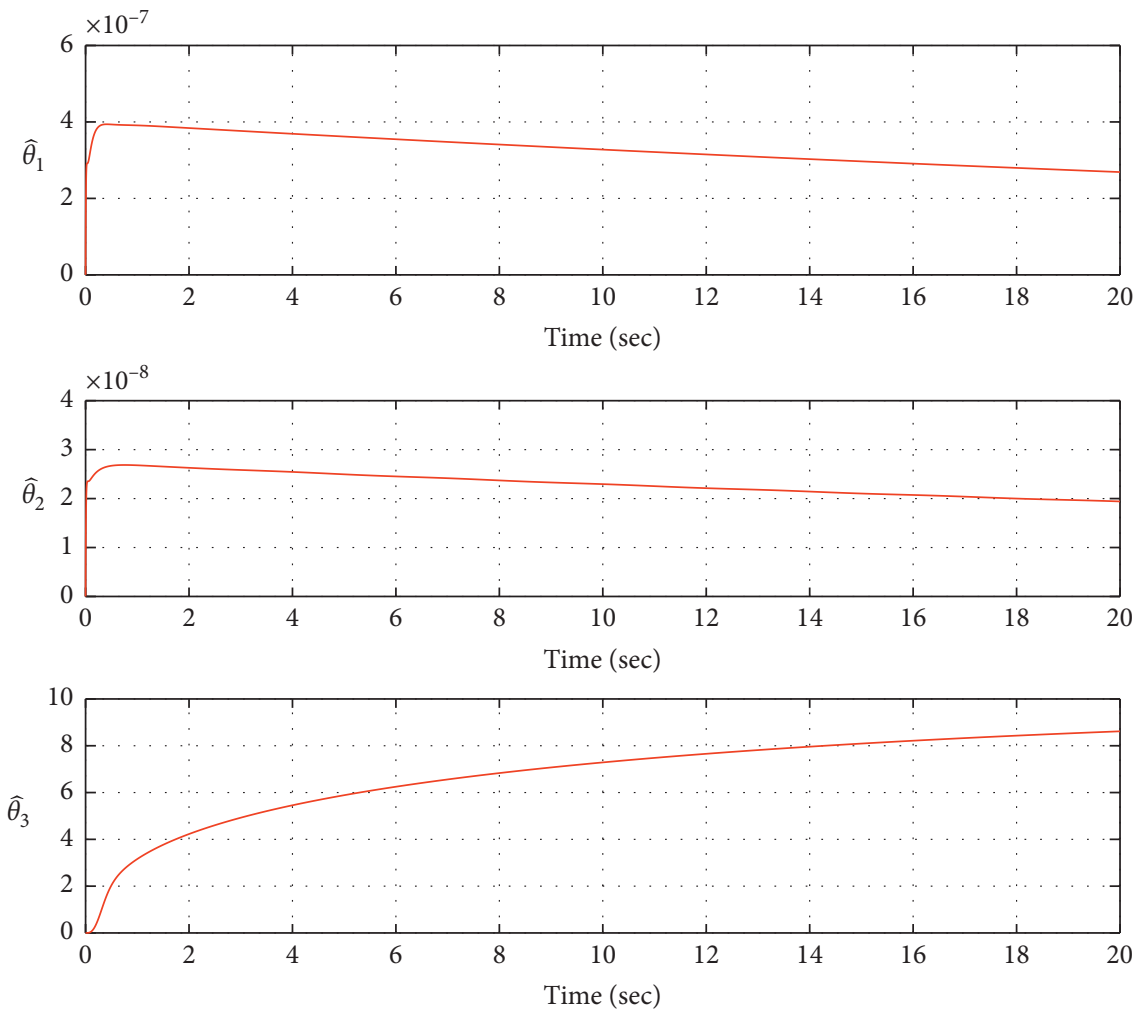

Figure 12: Adaptive parameters $\widehat{\theta}_{1}, \widehat{\theta}_{2}$, and $\widehat{\theta}_{3}$. 

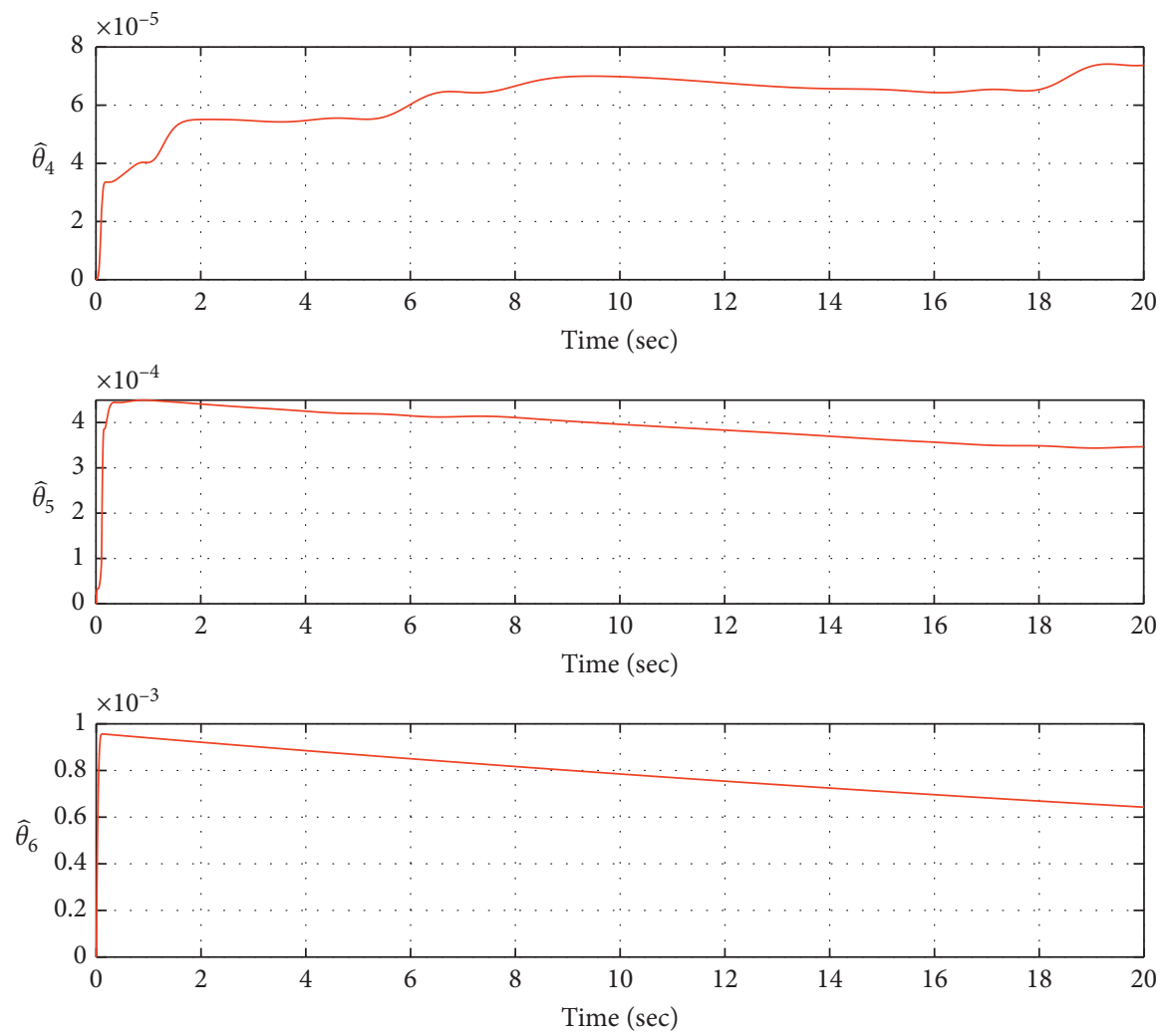

FIgUre 13: Adaptive parameters $\widehat{\theta}_{4}, \widehat{\theta}_{5}$, and $\widehat{\theta}_{6}$.
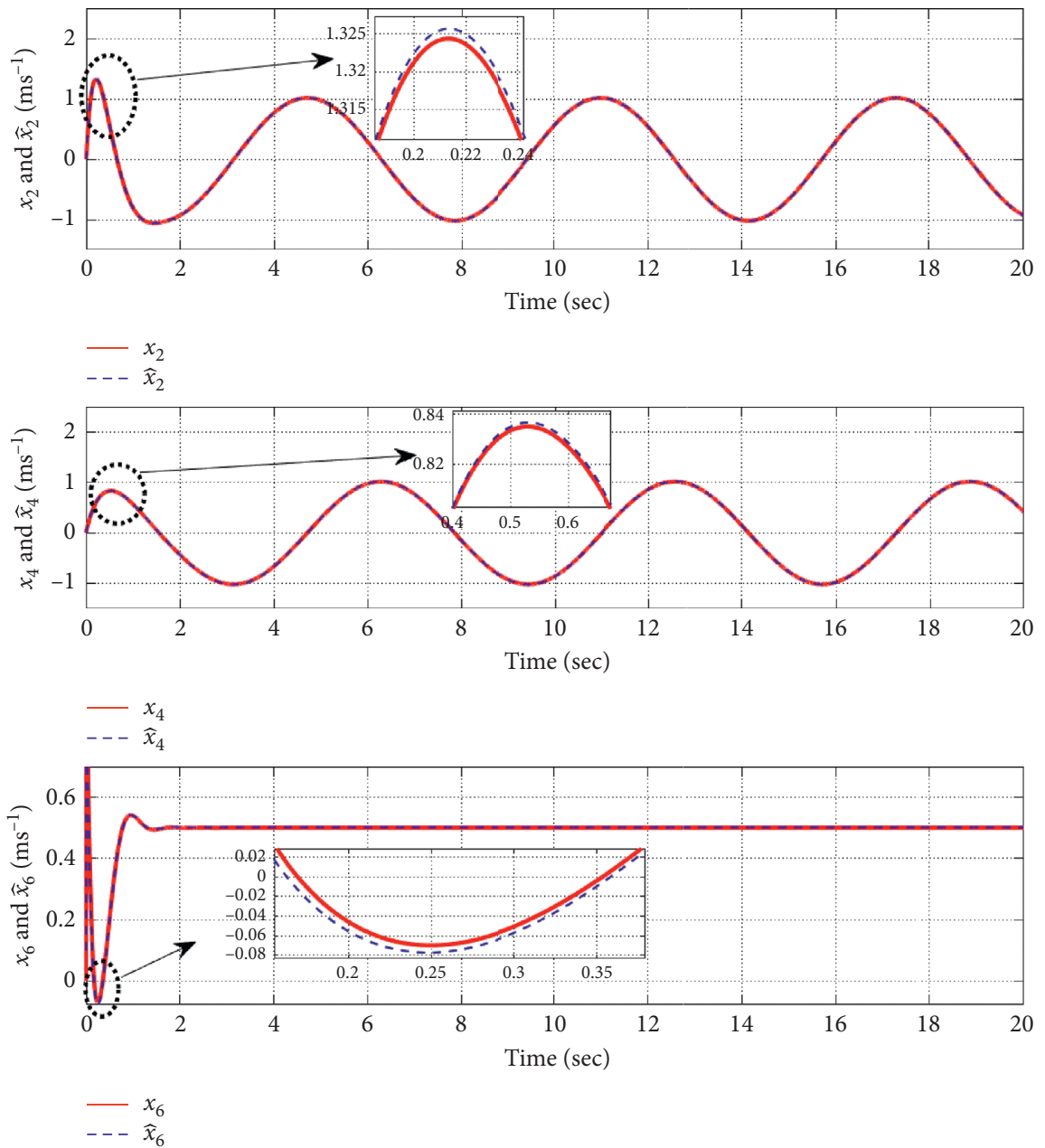

FIgURe 14: Actual value and estimated value. 

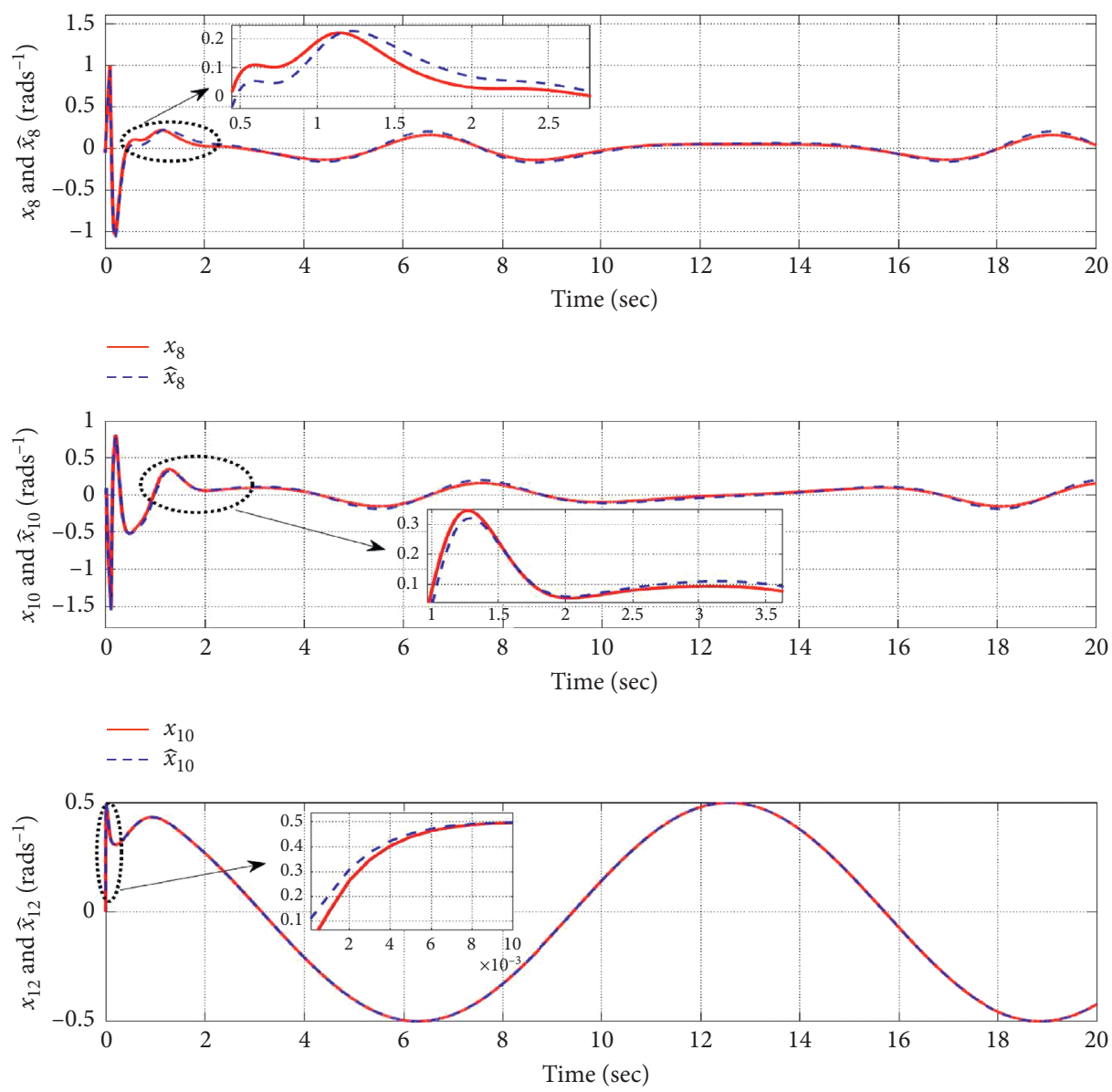

$-x_{12}$

FIGURE 15: Actual value and estimated value.

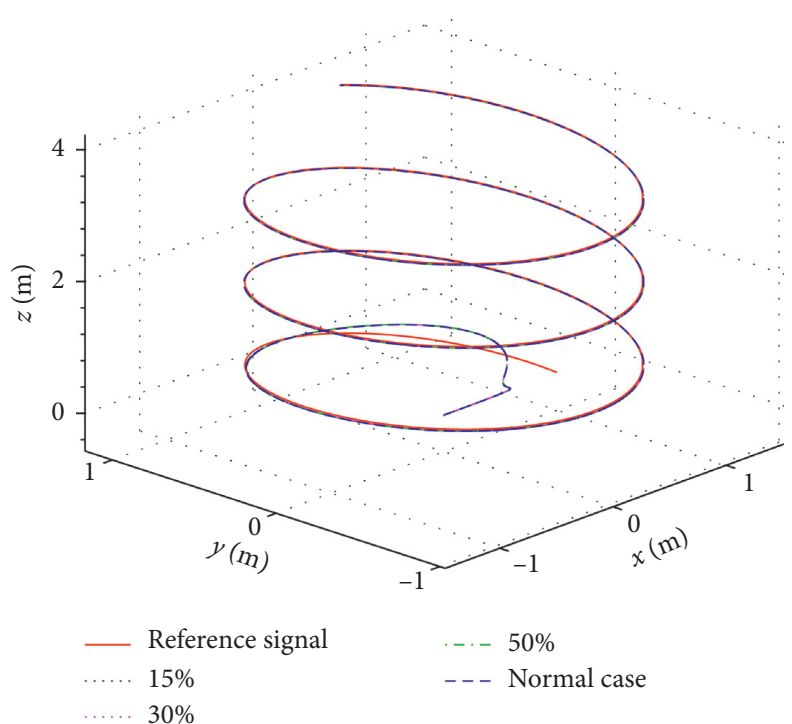

FIgURE 16: Space diagram of the position with normal, 15\%, 30\%, and 50\% parameter uncertainty. 

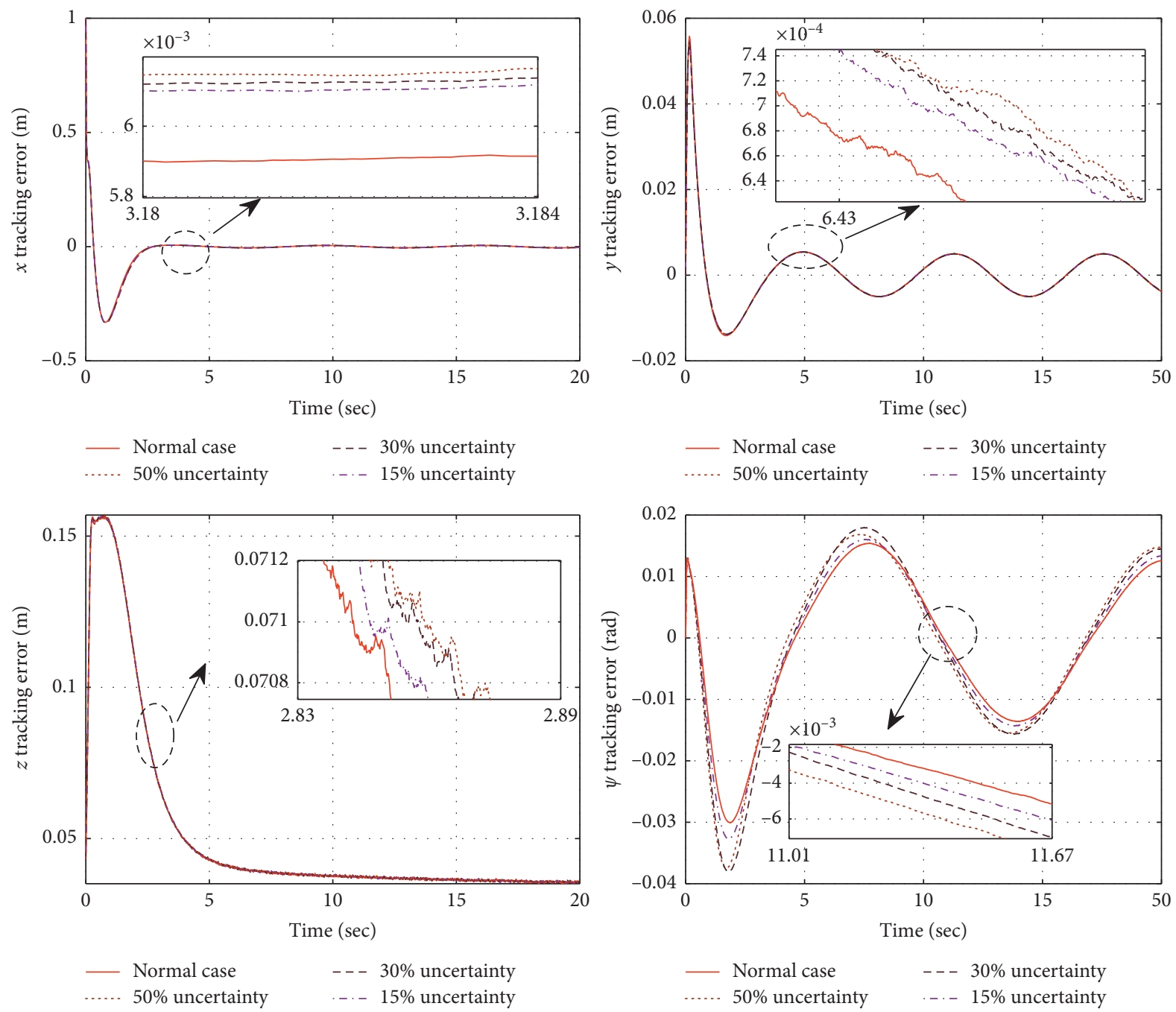

FIGURE 17: The tracking errors of different uncertainty cases added in the $z$ rotating axis.

TABLe 4: The MVTE and RMSVTE.

\begin{tabular}{cccccc}
\hline \multicolumn{2}{c}{ Kind of errors } & Normal case & Uncertainty $15 \%$ & Uncertainty $30 \%$ & Uncertainty $50 \%$ \\
\hline \multirow{4}{*}{ MVTE } & $x(\mathrm{~m})$ & $5.1254 e^{-03}$ & $5.5325 e^{-03}$ & $5.7471 e^{-03}$ & $5.9153 e^{-03}$ \\
& $y(\mathrm{~m})$ & $4.9051 e^{-03}$ & $5.2051 e^{-03}$ & $5.3593 e^{-03}$ & $5.5154 e^{-03}$ \\
& $z(\mathrm{~m})$ & $4.3216 e^{-02}$ & $4.4239 e^{-02}$ & $1.6869 e^{-02}$ & $4.5935 e^{-02}$ \\
\multirow{4}{*}{ RMSVTE } & $\psi(\mathrm{rad})$ & $1.5415 e^{-02}$ & $1.6041 e^{-02}$ & $4.1057 e^{-03}$ & $1.7956 e^{-02}$ \\
& $x(\mathrm{~m})$ & $3.6036 e^{-03}$ & $3.9049 e^{-03}$ & $3.5137 e^{-03}$ & $4.2059 e^{-03}$ \\
& $y(\mathrm{~m})$ & $3.4844 e^{-03}$ & $3.4966 e^{-03}$ & $3.7710 e^{-02}$ & $3.5714 e^{-03}$ \\
& $z(\mathrm{~m})$ & $3.7271 e^{-02}$ & $3.7414 e^{-02}$ & $1.1347 e^{-02}$ & $3.8117 e^{-02}$ \\
\hline
\end{tabular}

hown in Table 4. From Table 4, we can see that the proposed control scheme has strong robustness, and even the uncertainty in yaw rotary inertia is up to $50 \%$.

\section{Conclusion}

In this paper, an adaptive dynamic surface sliding-mode output feedback controller has been proposed for attitude and position control of a class of quadrotor UAVs with consideration of parametric uncertainties and disturbances.
By using the norm estimation approach, there is only one parameter which needs to be updated online at each design step regardless of the plant order and input-output dimension. Also, by introducing an error transformed function, the tracking performance of the quadrotor UAV has been achieved. The proposed control scheme can not only eliminate the problem of "explosion of complexity" existing in the backstepping control scheme but also improve the robustness of the system. The results of the hardware-inloop simulation validate the effectiveness of the proposed 
control algorithm. Furthermore, our future research work will focus on applying the control algorithm of this paper to the quadrotor UAV test platform for experimental verification.

\section{Appendix}

\section{A. The Procedures of Controller Design}

The controller design process.

Step 1. After error transformation, let $S_{1}$ given by (25) be the first error variable. Then, the derivative of $S_{1}$ can be expressed as

$$
\dot{S}_{1}=\eta_{1} \dot{\hat{x}}_{1}-\eta_{1} v_{1} .
$$

According to adaptive laws (T1.2), (A.1) can be rewritten as

$$
\dot{S}_{1}=\eta_{1}\left[\widehat{x}_{2}+k_{1}\left(x_{1}-\widehat{x}_{1}\right)\right]-\eta_{1} v_{1},
$$

which suggests that we choose the virtual control signal $\bar{x}_{2 d}$ as $\bar{x}_{2 d}=-k_{1}\left(x_{1}-\hat{x}_{1}\right)+v_{1}-S_{1} c_{1} / \eta_{1}$, where $c_{1}$ is a positive constant. Introduce a new state variable $x_{2 d}$, which can be obtained by the following first-order filter:

$$
\tau_{2} \dot{x}_{2 d}+x_{2 d}=\bar{x}_{2 d}, \quad x_{2 d}(0)=\bar{x}_{2 d}(0) .
$$

Define the error surface (T1.4), and the time derivative of $S_{2}$ is

$$
\dot{S}_{2}=\dot{\hat{x}}_{2}-\dot{x}_{2 d}=F_{1}\left(X_{1}\right)+\chi_{1}+k_{2}\left(x_{1}-\hat{x}_{1}\right)-\dot{x}_{2 d},
$$

where variables $\chi_{1}=\left(C_{x_{7}} S_{x_{9}} C_{x_{11}}+S_{x_{7}} S_{x_{11}}\right) U_{1}$ and $F_{1}\left(X_{1}\right)=$ $\hat{f}\left(\hat{x}_{2}\right), X_{1}=\hat{x}_{2}$ are introduced. Since $F_{1}\left(X_{1}\right)$ is unknown, we use FLSs to approximate the function $F_{1}\left(X_{1}\right)$ :

$$
F_{1}\left(X_{1}\right)=W_{1}^{* T} \xi_{1}\left(X_{1}\right)+\sigma_{1}^{*}, \quad\left|\sigma_{1}^{*}\right| \leq \varepsilon_{1},
$$

with respect to the unknown optimal weight vector in (A.5), define $\theta_{1}=\left\|W_{1}^{*}\right\|^{2}$, and since $\theta_{1}$ is unknown, let $\widehat{\theta}_{1}$ be the estimation of $\theta_{1}$ and $\widetilde{\theta}_{1}=\theta_{1}-\widehat{\theta}_{1}$. Choosing the following proper sliding surface $\sigma_{s 1}=S_{2}$, consider the first Lyapunov function:

$$
\Gamma_{1}=\frac{1}{2}\left(S_{1}^{2}+S_{2}^{2}+\widetilde{\theta}_{1}^{2}\right),
$$

where the differential of Lyapunov Function $\Gamma_{1}$ can be found as follows:

$$
\begin{aligned}
\dot{\Gamma}_{1}= & S_{1} \dot{S}_{1}+S_{2} \dot{S}_{2}-\widetilde{\theta}_{1} \dot{\hat{\theta}}_{1} \\
= & -c_{1} S_{1}^{2}+S_{1} \eta_{1}\left(\hat{x}_{2}-\bar{x}_{2 d}\right)+S_{2} W_{1}^{* T} \xi_{1}\left(X_{1}\right)+S_{2} \sigma_{1}^{*}-\frac{1}{2} S_{2}^{2} \\
& +S_{2} \chi_{1}+S_{2} k_{2}\left(x_{1}-\widehat{x}_{1}\right)-S_{2} \dot{x}_{2 d}-\widetilde{\theta}_{1} \dot{\hat{\theta}}_{1} .
\end{aligned}
$$

Using Young's inequality, it can be verified that

$$
\begin{aligned}
S_{2} W_{1}^{* T} \xi_{1}\left(X_{1}\right) & \leq \frac{1}{2} S_{2}^{2}\left\|W_{1}^{*}\right\|^{2} \xi_{1}^{T}\left(X_{1}\right) \xi_{1}\left(X_{1}\right)+\frac{1}{2} \\
& \leq \frac{1}{2} S_{2}^{2} \theta_{1} \xi_{1}^{T}\left(X_{1}\right) \xi_{1}\left(X_{1}\right)+\frac{1}{2}, \\
S_{2} \sigma_{1}^{*} & \leq \frac{1}{2} S_{2}^{2}+\frac{1}{2} \varepsilon_{1}^{2} .
\end{aligned}
$$

Then, (A.7) can be rewritten as

$$
\begin{aligned}
\dot{\Gamma}_{1} \leq & -c_{1} S_{1}^{2}+\frac{1}{2} S_{2}^{2} \hat{\theta}_{1} \xi_{1}^{T}\left(X_{1}\right) \xi_{1}\left(X_{1}\right)+S_{2} \chi_{1}+S_{2} k_{2}\left(x_{1}-\widehat{x}_{1}\right)-S_{2} \dot{x}_{2 d} \\
& +\frac{1}{2}+\frac{1}{2} \varepsilon_{1}^{2}-\tilde{\theta}_{1}\left[\dot{\hat{\theta}}_{1}-\frac{1}{2} S_{2}^{2} \xi_{1}^{T}\left(X_{1}\right) \xi_{1}\left(X_{1}\right)\right] \\
& +S_{1} \eta_{1}\left(\hat{x}_{2}-\bar{x}_{2 d}\right)+\frac{1}{2} S_{2}^{2} .
\end{aligned}
$$

The stabilization of $\Gamma_{1}$ can be obtained by designing the virtual control (T1.5) and the adaptation law (T1.6), where $c_{2}, \mu_{1}$, and $\lambda_{1}$ are the positive constants and $\varepsilon_{1}$ is an arbitrarily small positive constant. For the external disturbance $d_{t 1}$ encountered in the quadrotor flight process, the sliding surface is added to maintain system stability with $\mu_{1} \geq\left|d_{t 1}\right|$. Substituting (T1.5) and (T1.6) into (A.9), we get

$$
\dot{\Gamma}_{1} \leq-c_{1} S_{1}^{2}+S_{1} \eta_{1}\left(\widehat{x}_{2}-\bar{x}_{2 d}\right)-\left(c_{2}-\frac{1}{2}\right) S_{2}^{2}+\frac{1}{2}+\frac{1}{2} \varepsilon_{1}^{2}+\lambda_{1} \tilde{\theta}_{1} \widehat{\theta}_{1} .
$$

Similar design procedures can be used to design adaptive DSC sliding-mode laws for trajectory tracking control of $y$ axis position $\left(x_{3}\right)$ and $z$ axis position $\left(x_{5}\right)$. Introduce two variables $\chi_{2}=\left(C_{x_{7}} S_{x_{9}} S_{x_{11}}-S_{x_{7}} C_{x_{11}}\right) U_{1} \quad$ and $\chi_{3}=\left(C_{x_{7}} C_{x_{9}}\right) U_{1}$. The corresponding control laws and adaptive laws are designed as follows.

Step 2. Let $S_{3}$ given by (26) be the second error variable. Then, the derivative of $S_{3}$ can be expressed as

$$
\dot{S}_{3}=\eta_{2} \dot{\hat{x}}_{3}-\eta_{2} v_{2} \text {. }
$$

According to (29) and (T1.8), (A.11) can be rewritten as

$$
\dot{S}_{3}=\eta_{2}\left[\widehat{x}_{4}+k_{1}\left(x_{3}-\hat{x}_{3}\right)\right]-\eta_{2} v_{2},
$$

choosing the virtual control signal $\bar{x}_{4 d}=-k_{1}\left(x_{3}-\hat{x}_{3}\right)+v_{2}-S_{3} c_{3} / \eta_{2}$, where $c_{3}$ is a positive constant. Introduce a new state variable $x_{4 d}$, which can be obtained by the following first-order filter:

$$
\tau_{4} \dot{x}_{4 d}+x_{4 d}=\bar{x}_{4 d}, \quad x_{4 d}(0)=\bar{x}_{4 d}(0) .
$$

Define the error surface (T1.10), and the time derivative of $S_{4}$ is

$$
\dot{S}_{4}=\dot{\hat{x}}_{4}-\dot{x}_{4 d}=F_{2}\left(X_{2}\right)+\chi_{2}+k_{2}\left(x_{3}-\widehat{x}_{3}\right)-\dot{x}_{4 d},
$$

where $F_{2}\left(X_{2}\right)=\hat{f}\left(\hat{x}_{4}\right), X_{2}=\hat{x}_{4}$. FLSs are used to approximate the unknown function $F_{2}\left(X_{2}\right)$ : 


$$
F_{2}\left(X_{2}\right)=W_{2}^{* T} \xi_{2}\left(X_{2}\right)+\sigma_{2}^{*}, \quad\left|\sigma_{2}^{*}\right| \leq \varepsilon_{2},
$$

with respect to the unknown optimal weight vector in (A.15), define $\theta_{2}=\left\|W_{2}^{*}\right\|^{2}$, and since $\theta_{2}$ is unknown, let $\widehat{\theta}_{2}$ be the estimation of $\theta_{2}$ and $\widetilde{\theta}_{2}=\theta_{2}-\widehat{\theta}_{2}$. Choosing the following proper sliding surface $\sigma_{s 2}=S_{4}$, consider the second Lyapunov function:

$$
\Gamma_{2}=\frac{1}{2}\left(S_{3}^{2}+S_{4}^{2}+\widetilde{\theta}_{2}^{2}\right) .
$$

Differentiating $\Gamma_{2}$, we obtain

$$
\begin{aligned}
\dot{\Gamma}_{2}= & S_{3} \dot{S}_{3}+S_{4} \dot{S}_{4}-\tilde{\theta}_{2} \dot{\hat{\theta}}_{2} \\
= & -c_{3} S_{3}^{2}+S_{3} \eta_{2}\left(\hat{x}_{4}-\bar{x}_{4 d}\right)+S_{4} W_{2}^{* T} \xi_{2}\left(X_{2}\right)+S_{4} \sigma_{2}^{*}-\frac{1}{2} S_{4}^{2} \\
& +S_{4} \chi_{2}+S_{4} k_{2}\left(x_{3}-\widehat{x}_{3}\right)-S_{4} \dot{x}_{4 d}-\tilde{\theta}_{2} \dot{\hat{\theta}}_{2} .
\end{aligned}
$$

Using Young's inequality, it can be verified that

$$
\begin{aligned}
S_{4} W_{2}^{* T} \xi_{2}\left(X_{2}\right) & \leq \frac{1}{2} S_{4}^{2}\left\|W_{2}^{*}\right\|^{2} \xi_{2}^{T}\left(X_{2}\right) \xi_{2}\left(X_{2}\right)+\frac{1}{2} \\
& \leq \frac{1}{2} S_{4}^{2} \theta_{1} \xi_{2}^{T}\left(X_{2}\right) \xi_{2}\left(X_{2}\right)+\frac{1}{2}, \\
S_{4} \sigma_{2}^{*} & \leq \frac{1}{2} S_{4}^{2}+\frac{1}{2} \varepsilon_{2}^{2} .
\end{aligned}
$$

Then, (A.17) can be rewritten as

$$
\begin{aligned}
\dot{\Gamma}_{2} \leq & -c_{3} S_{3}^{2}+\frac{1}{2} S_{4}^{2} \widehat{\theta}_{2} \xi_{2}^{T}\left(X_{2}\right) \xi_{2}\left(X_{2}\right)+S_{4} \chi_{2} \\
& +S_{4} k_{2}\left(x_{3}-\widehat{x}_{3}\right)-S_{4} \dot{x}_{4 d} \\
& +\frac{1}{2}+\frac{1}{2} \varepsilon_{2}^{2}-\widetilde{\theta}_{2}\left[\dot{\hat{\theta}}_{2}-\frac{1}{2} S_{4}^{2} \xi_{2}^{T}\left(X_{2}\right) \xi_{2}\left(X_{2}\right)\right] \\
& +\frac{1}{2} S_{4}^{2}+S_{3} \eta_{2}\left(\hat{x}_{4}-\bar{x}_{4 d}\right) .
\end{aligned}
$$

The stabilization of $\Gamma_{2}$ can be obtained by designing the virtual control (T1.11) and the adaptation law (T1.12), where $c_{4}, \mu_{2}$, and $\lambda_{2}$ are the positive constants and $\varepsilon_{2}$ is an arbitrarily small positive constant. For the external disturbance $d_{t}$ encountered in the quadrotor flight process, the sliding surface is added to maintain system stability with $\mu_{1} \geq\left|d_{t 2}\right|$. Substituting (T1.11) and (T1.12) into (A.20), we get

$$
\dot{\Gamma}_{2} \leq-c_{3} S_{3}^{2}+S_{3} \eta_{2}\left(\widehat{x}_{4}-\bar{x}_{4 d}\right)-\left(c_{4}-\frac{1}{2}\right) S_{4}^{2}+\frac{1}{2}+\frac{1}{2} \varepsilon_{2}^{2}+\lambda_{2} \widetilde{\theta}_{2} \widehat{\theta}_{2}
$$

Step 3. Let $S_{5}$ given by (26) be the third error variable. Then, the derivative of $S_{5}$ can be expressed as

$$
\dot{S}_{5}=\eta_{3} \dot{\bar{x}}_{5}-\eta_{3} v_{3} \text {. }
$$

According to (29) and adaptive laws (T1.14), (A.21) can be rewritten as

$$
\dot{S}_{5}=\eta_{3}\left[\hat{x}_{6}+k_{1}\left(x_{5}-\hat{x}_{5}\right)\right]-\eta_{3} v_{3},
$$

and the virtual control signal can be chosen as $\bar{x}_{6 d}$ as $\bar{x}_{6 d}=$ $-k_{1}\left(x_{5}-\hat{x}_{5}\right)+v_{3}-S_{5} c_{5} / \eta_{3}$ with $c_{5}$ being a positive constant. Introduce a new state variable $x_{6 d}$, which can be obtained by the following first-order filter:

$$
\tau_{6} \dot{x}_{6 d}+x_{6 d}=\bar{x}_{6 d}, \quad x_{6 d}(0)=\bar{x}_{6 d}(0) .
$$

Define the error surface (T1.16), and the time derivative of $S_{6}$ is

$$
\dot{S}_{6}=\dot{\hat{x}}_{6}-\dot{x}_{6 d}=F_{3}\left(X_{3}\right)+\chi_{3}+k_{2}\left(x_{5}-\widehat{x}_{5}\right)-\dot{x}_{6 d},
$$

where $F_{3}\left(X_{3}\right)=\hat{f}\left(\hat{x}_{6}\right), X_{3}=\hat{x}_{6}$. Utilizing FLSs to approximate the unknown function $F_{3}\left(X_{3}\right)$, we obtain

$$
F_{3}\left(X_{3}\right)=W_{3}^{* T} \xi_{3}\left(X_{3}\right)+\sigma_{3}^{*}, \quad\left|\sigma_{3}^{*}\right| \leq \varepsilon_{3} .
$$

Define $\theta_{3}=\left\|W_{3}^{*}\right\|^{2}$, and let $\widehat{\theta}_{3}$ be the estimation of $\theta_{3}$ and $\widetilde{\theta}_{3}=\theta_{3}-\widehat{\theta}_{3}$. Choosing the following proper sliding surface $\sigma_{s 3}=S_{6}$, consider the third Lyapunov function:

$$
\Gamma_{3}=\frac{1}{2}\left(S_{5}^{2}+S_{6}^{2}+\tilde{\theta}_{3}^{2}\right) \text {. }
$$

The differentiation of $\Gamma_{3}$ is as follows:

$$
\begin{aligned}
\dot{\Gamma}_{3}= & S_{5} \dot{S}_{5}+S_{6} \dot{S}_{6}-\widetilde{\theta}_{3} \dot{\hat{\theta}}_{3} \\
= & -c_{5} S_{5}^{2}+S_{5} \eta_{3}\left(\hat{x}_{6}-\bar{x}_{6 d}\right)+S_{6} W_{3}^{* T} \xi_{3}\left(X_{3}\right)+S_{6} \sigma_{3}^{*}-\frac{1}{2} S_{6}^{2} \\
& +S_{6} \chi_{3}+S_{6} k_{2}\left(x_{5}-\widehat{x}_{5}\right)-S_{6} \dot{x}_{6 d}-\tilde{\theta}_{3} \dot{\hat{\theta}}_{3} .
\end{aligned}
$$

Using Young's inequality, it can be verified that

$$
\begin{aligned}
S_{6} W_{3}^{* T} \xi_{3}\left(X_{3}\right) & \leq \frac{1}{2} S_{6}^{2}\left\|W_{3}^{*}\right\|^{2} \xi_{3}^{T}\left(X_{3}\right) \xi_{3}\left(X_{3}\right)+\frac{1}{2} \\
& \leq \frac{1}{2} S_{6}^{2} \theta_{3} \xi_{3}^{T}\left(X_{3}\right) \xi_{3}\left(X_{3}\right)+\frac{1}{2}, \\
S_{6} \sigma_{3}^{*} & \leq \frac{1}{2} S_{6}^{2}+\frac{1}{2} \varepsilon_{3}^{2} .
\end{aligned}
$$

Then, (A.27) can be rewritten as

$$
\begin{aligned}
\dot{\Gamma}_{3} \leq & -c_{5} S_{5}^{2}+\frac{1}{2} S_{6}^{2} \widehat{\theta}_{3} \xi_{3}^{T}\left(X_{3}\right) \xi_{3}\left(X_{3}\right)+S_{6} \chi_{3} \\
& +S_{6} k_{2}\left(x_{5}-\widehat{x}_{5}\right)-S_{6} \dot{x}_{6 d} \\
& +\frac{1}{2}+\frac{1}{2} \varepsilon_{3}^{2}-\tilde{\theta}_{3}\left[\dot{\hat{\theta}}_{3}-\frac{1}{2} S_{6}^{2} \xi_{3}^{T}\left(X_{3}\right) \xi_{3}\left(X_{3}\right)\right]+\frac{1}{2} S_{6}^{2} \\
& +S_{5} \eta_{3}\left(\hat{x}_{6}-\bar{x}_{6 d}\right) .
\end{aligned}
$$


The stabilization of $\Gamma_{3}$ can be obtained by designing the virtual control (T1.17) and the adaptive law (T1.18), where $c_{6}, \mu_{3}$, and $\lambda_{3}$ are the positive constants and $\varepsilon_{3}$ is an arbitrarily small positive constant. For the external disturbance $d_{t 3}$, the sliding surface is added to maintain system stability with $\mu_{3} \geq\left|d_{t 3}\right|$. Substituting (T1.17) and (T1.18) into (A.29), we get

$$
\dot{\Gamma}_{3} \leq-c_{5} S_{5}^{2}+S_{5} \eta_{3}\left(\widehat{x}_{6}-\bar{x}_{6 d}\right)-\left(c_{6}-\frac{1}{2}\right) S_{6}^{2}+\frac{1}{2}+\frac{1}{2} \varepsilon_{3}^{2}+\lambda_{3} \widetilde{\theta}_{3} \widehat{\theta}_{3} .
$$

By associating $\chi_{1}, \chi_{2}$, and $\chi_{3}$, the virtual controllers are obtained as

$$
\begin{aligned}
& \chi_{1}=\left(C_{x_{7}} S_{x_{9}} C_{x_{11}}+S_{x_{7}} S_{x_{11}}\right) U_{1}, \\
& \chi_{2}=\left(C_{x_{7}} S_{x_{9}} S_{x_{11}}-S_{x_{7}} C_{x_{11}}\right) U_{1}, \\
& \chi_{3}=\left(C_{x_{7}} C_{x_{9}}\right) U_{1} .
\end{aligned}
$$

Notably, (A.31) has four degrees of freedom, namely, $x_{7}$, $x_{9}, x_{11}$, and $U_{1}$. We consider the reference trajectory for yaw angle $x_{11 d}$, which is usually given in advance, and the corresponding DSC sliding-mode law $U_{4}$ is directly designed in the next section to ensure the rapid convergence of $x_{11}$ to $x_{11 d}$. Thus, we regarded $x_{11}$ as known that can be replaced by $x_{11 d}$ in the controller, and the degrees of freedom in (A.31) is reduced so that $x_{7}, x_{9}$, and $U_{1}$ can be solved. The programs are as follows:

$$
\left\{\begin{array}{l}
\bar{x}_{7 d}=\arctan \left(C_{x_{9}} \frac{\beta \chi_{1}-\alpha \chi_{2}}{\chi_{3}}\right) \\
\bar{x}_{9 d}=\arctan \left(\frac{\alpha \chi_{1}+\beta \chi_{2}}{\chi_{3}}\right) \\
U_{1}=\frac{\chi_{3}}{C_{x_{7}} C_{x_{9}}}
\end{array}\right.
$$

where $U_{1}$ is one of the ultimate control laws, and in addition, $\alpha=\cos \left(x_{11 d}\right)$ and $\beta=\sin \left(x_{11 d}\right)$. Introduce two new state variables $x_{7 d}$ and $x_{9 d}$, which can be obtained by the following first-order filters:

$$
\begin{array}{ll}
\tau_{7} \dot{x}_{7 d}+x_{7 d}=\bar{x}_{7 d}, & x_{7 d}(0)=\bar{x}_{7 d}(0), \\
\tau_{9} \dot{x}_{9 d}+x_{9 d}=\bar{x}_{9 d}, & x_{9 d}(0)=\bar{x}_{9 d}(0) .
\end{array}
$$

For attitude trajectory tracking control, by taking $\left\{x_{7 d}, x_{9 d}, x_{11 d}\right\}$ as the desired attitude trajectory, the design of control laws contains three steps. The attitude dynamic system can be extracted as follows:

$$
\left\{\begin{array}{l}
\dot{x}_{7}=x_{8}, \\
\dot{x}_{8}=a_{4} x_{10} x_{12}+a_{5} x_{10} \Omega-a_{6} x_{8}+U_{2}, \\
\dot{x}_{9}=x_{10} \\
\dot{x}_{10}=a_{7} x_{8} x_{12}+a_{8} x_{8} \Omega-a_{9} x_{10}+U_{3}, \\
\dot{x}_{11}=x_{12} \\
\dot{x}_{12}=a_{10} x_{8} x_{10}-a_{11} x_{12}+U_{4} .
\end{array}\right.
$$

Step 4. Let $S_{7}$ given by (26) be the fourth error variable. Then, the derivative of $S_{7}$ can be expressed as

$$
\dot{S}_{7}=\eta_{4} \dot{\hat{x}}_{7}-\eta_{4} v_{4} .
$$

According to (29) and adaptive laws (T2.2) and (T2.3), where $\quad \widehat{f}\left(\hat{x}_{8}, \widehat{x}_{10}, \widehat{x}_{12}\right)=\Delta a_{4} \hat{x}_{10} \widehat{x}_{12}+\Delta a_{5} \widehat{x}_{10} \Omega-\Delta a_{6} \widehat{x}_{8}$, (A.35) can be rewritten as

$$
\dot{S}_{7}=\eta_{4}\left[\hat{x}_{8}+k_{1}\left(x_{7}-\hat{x}_{7}\right)\right]-\eta_{4} v_{4},
$$

which suggests that we choose the virtual control signal $\bar{x}_{8 d}$ as $\bar{x}_{8 d}=-k_{1}\left(x_{7}-\hat{x}_{7}\right)+v_{4}-S_{7} c_{7} / \eta_{4}$, where $c_{7}$ is a positive constant. Introduce a new state variable $x_{8 d}$, which can be obtained by the following first-order filter:

$$
\tau_{8} \dot{x}_{8 d}+x_{8 d}=\bar{x}_{8 d}, \quad x_{8 d}(0)=\bar{x}_{8 d}(0) .
$$

Define the error surface (T2.5), and the time derivative of $S_{8}$ is

$$
\begin{aligned}
\dot{S}_{8}= & \dot{x}_{8}-\dot{x}_{8 d}=a_{4 N} \widehat{x}_{10} \widehat{x}_{12}+a_{5 N} \widehat{x}_{10} \Omega-a_{6 N} \widehat{x}_{8} \\
& +F_{4}\left(X_{4}\right)+U_{2}+k_{2}\left(x_{7}-\widehat{x}_{7}\right)-\dot{x}_{8 d},
\end{aligned}
$$

where $F_{4}\left(X_{4}\right)=\hat{f}\left(\widehat{x}_{8}, \hat{x}_{10}, \hat{x}_{12}\right), X_{4}=\left[\hat{x}_{8}, \widehat{x}_{10}, \widehat{x}_{12}\right]^{T}$. Utilizing FLSs to approximate the unknown function $F_{4}\left(X_{4}\right)$, we obtain

$$
F_{4}\left(X_{4}\right)=W_{4}^{* T} \xi_{4}\left(X_{4}\right)+\sigma_{4}^{*}, \quad\left|\sigma_{4}^{*}\right| \leq \varepsilon_{4} .
$$

Define $\theta_{4}=\left\|W_{4}^{*}\right\|^{2}$, and let $\widehat{\theta}_{4}$ be the estimation of $\theta_{4}$ and $\tilde{\theta}_{4}=\theta_{4}-\widehat{\theta}_{4}$. Choosing the following proper sliding surface $\sigma_{s 4}=S_{8}$, consider the following Lyapunov function:

$$
\Gamma_{4}=\frac{1}{2}\left(S_{7}^{2}+S_{8}^{2}+\widetilde{\theta}_{4}^{2}\right),
$$

where the differential of Lyapunov function $\Gamma_{4}$ can be found as follows:

$$
\begin{aligned}
\dot{\Gamma}_{4}= & S_{7} \dot{S}_{7}+S_{8} \dot{S}_{8}-\widetilde{\theta}_{4} \dot{\hat{\theta}}_{4} \\
= & -c_{7} S_{7}^{2}+S_{7} \eta_{4}\left(\hat{x}_{8}-\bar{x}_{8 d}\right)+S_{8} W_{4}^{* T} \xi_{4}\left(X_{4}\right)+S_{8} \sigma_{4}^{*}-\frac{1}{2} S_{8}^{2} \\
& +S_{8} U_{2}+S_{8} k_{2}\left(x_{7}-\widehat{x}_{7}\right)-S_{8} \dot{x}_{8 d}+\widetilde{\theta}_{4} \dot{\hat{\theta}}_{4} .
\end{aligned}
$$

Using Young's inequality, it can be verified that

$$
\begin{aligned}
S_{8} W_{4}^{* T} \xi_{4}\left(X_{4}\right) & \leq \frac{1}{2} S_{8}^{2}\left\|W_{4}^{*}\right\|^{2} \xi_{4}^{T}\left(X_{4}\right) \xi_{4}\left(X_{4}\right)+\frac{1}{2} \\
& \leq \frac{1}{2} S_{8}^{2} \theta_{4} \xi_{4}^{T}\left(X_{4}\right) \xi_{4}\left(X_{4}\right)+\frac{1}{2}, \\
S_{8} \sigma_{4}^{*} & \leq \frac{1}{2} S_{8}^{2}+\frac{1}{2} \varepsilon_{4}^{2} .
\end{aligned}
$$




$$
\begin{aligned}
\dot{\Gamma}_{4} \leq & -c_{7} S_{7}^{2}+\frac{1}{2} S_{8}^{2} \widehat{\theta}_{4} \xi_{4}^{T}\left(X_{4}\right) \xi_{4}\left(X_{4}\right)+S_{8}\left(a_{4} N \hat{x}_{10} \widehat{x}_{12}\right. \\
& \left.+a_{5 N} \widehat{x}_{10} \Omega-a_{6 N} \widehat{x}_{8}\right)+S_{8} U_{2}+S_{8} k_{2}\left(x_{7}-\widehat{x}_{7}\right) \\
& -S_{8} \dot{x}_{8 d}+\frac{1}{2}+\frac{1}{2} \varepsilon_{4}^{2}+\frac{1}{2} S_{8}^{2} \\
& +S_{7} \eta_{4}\left(\widehat{x}_{8}-\bar{x}_{8 d}\right) \\
& -\widetilde{\theta}_{4}\left[\dot{\hat{\theta}}_{4}-\frac{1}{2} S_{8}^{2} \xi_{4}^{T}\left(X_{4}\right) \xi_{4}\left(X_{4}\right)\right] .
\end{aligned}
$$

$$
\begin{aligned}
\dot{\Gamma}_{5} \leq & -c_{9} S_{9}^{2}+S_{9} \eta_{5}\left(\widehat{x}_{10}-\bar{x}_{10 d}\right)-\left(c_{10}-\frac{1}{2}\right) S_{10}^{2}+\frac{1}{2} \\
& +\frac{1}{2} \varepsilon_{5}^{2}+\lambda_{5} \widetilde{\theta}_{5} \widehat{\theta}_{5}, \\
\dot{\Gamma}_{6} \leq & -c_{11} S_{11}^{2}+S_{11} \eta_{6}\left(\widehat{x}_{12}-\bar{x}_{12 d}\right)-\left(c_{12}-\frac{1}{2}\right) S_{12}^{2}+\frac{1}{2} \\
& +\frac{1}{2} \varepsilon_{6}^{2}+\lambda_{6} \widetilde{\theta}_{6} \widehat{\theta}_{6} .
\end{aligned}
$$

The stabilization of $\Gamma_{4}$ can be obtained by designing the virtual control (T2.6) and the adaptation law (T2.7), where $c_{8}, \mu_{4}$, and $\lambda_{4}$ are positive constants and $\varepsilon_{1}$ is an arbitrarily small positive constant. For the external disturbance $d_{t 4}$ encountered in the quadrotor flight process, the sliding surface is added to maintain system stability with $\mu_{4} \geq\left|d_{t 4}\right|$. Substituting (T2.6) and (T2.7) into (A.43), we get

$$
\dot{\Gamma}_{4} \leq-c_{7} S_{7}^{2}+S_{7} \eta_{4}\left(\widehat{x}_{8}-\bar{x}_{8 d}\right)-\left(c_{8}-\frac{1}{2}\right) S_{8}^{2}+\frac{1}{2}+\frac{1}{2} \varepsilon_{4}^{2}+\lambda_{4} \widetilde{\theta}_{4} \widehat{\theta}_{4}
$$

Similarly, the adaptive DSC sliding-mode laws for trajectory tracking control of pitch angle $\left(x_{9}\right)$ and yaw angle $\left(x_{11}\right)$ can be designed as (T2.13), (T2.20), (T2.14), and (T2.21), where $S_{10}=\widehat{x}_{10}-x_{10 d}, S_{12}=\widehat{x}_{12}-x_{12 d}, \quad X_{5}=$ $\left[\hat{x}_{8}, \hat{x}_{10}, \hat{x}_{12}\right]^{T}, X_{6}=\left[\hat{x}_{8}, \hat{x}_{10}, \hat{x}_{12}\right]^{T}, x_{10 d}$ and $x_{12 d}$ are the output of each first-order filter, and $c_{9}, c_{10}, c_{11}, c_{12}, \lambda_{5}, \lambda_{6}, \mu_{5}$, and $\mu_{6}$ are the positive constants.

The derivative of Lyapunov candidate for pitch angle $\left(x_{9}\right)$ and yaw angle $\left(x_{11}\right)$ are designed as follows:

\section{B. Proof of Theorem 1}

Taking the time derivative of $\Gamma_{i},(i=1, \ldots, 6)$ and combing (38) yields

$$
\begin{aligned}
\dot{\Gamma}_{i} \leq & -c_{(2 i-1)} S_{(2 i-1)}^{2}+S_{(2 i-1)} \eta_{i}\left(S_{2 i}+y_{2 i}\right)-\left(c_{2 i}-\frac{1}{2}\right) S_{2 i}^{2} \\
& +\frac{1}{2}+\frac{1}{2} \varepsilon_{i}^{2}+\lambda_{i} \widetilde{\theta}_{i} \widehat{\theta}_{i}
\end{aligned}
$$$$
y_{2 i} \dot{y}_{2 i} \leq-\frac{y_{2 i}^{2}}{\tau_{2 i}}+B_{2 i}\left|y_{2 i}\right|, \quad i=1, \ldots, 6 \text {, }
$$$$
y_{7} \dot{y}_{7} \leq-\frac{y_{7}^{2}}{\tau_{7}}+B_{7}\left|y_{7}\right| \text {, }
$$$$
y_{9} \dot{y}_{9} \leq-\frac{y_{9}^{2}}{\tau_{9}}+B_{9}\left|y_{9}\right| \text {. }
$$

Consider the sets

$$
\begin{aligned}
\Upsilon_{1}:= & \left\{\left(x_{d}, \dot{x}_{d}, x_{d}, y_{d}, \dot{y}_{d}, \ddot{y}_{d}, z_{d}, \dot{z}_{d}, z_{d}, \psi_{d}, \dot{\psi}_{d}, \psi_{d}\right): x_{d}^{2}+\dot{x}_{d}^{2}+x_{d}^{2}+y_{d}^{2}+\dot{y}_{d}^{2}+y_{d}^{2}\right. \\
& \left.+z_{d}^{2}+\dot{z}_{d}^{2}+z_{d}^{2}+\psi_{d}^{2}+\dot{\psi}_{d}^{2}+\psi_{d}^{2}\right\} \leq B_{0}, \\
\Upsilon_{2}:= & \left\{\sum_{i=1}^{6} \Gamma_{i}+\sum_{i=1}^{6} \frac{1}{2} y_{2 i}^{2}+\frac{1}{2} y_{7}^{2}+\frac{1}{2} y_{9}^{2} \leq p\right\}
\end{aligned}
$$

where $\Upsilon_{1} \times \Upsilon_{2}$ is also in a compact set. Then, the continuous functions $B_{2 i}(\cdot),(i=1, \ldots, 6), B_{7}, B_{9}$ have maximums on $\Upsilon_{1} \times \Upsilon_{2}$, say, $M_{i}(i=1, \ldots, 6), M_{7}, M_{9}$. Thus, 


$$
\begin{aligned}
& y_{2 i} y_{2 i}^{2} \leq-\frac{y_{2 i}^{2}}{\tau_{2 i}}+\frac{M_{i}^{2}\left|y_{2 i}^{2}\right|}{2 \varrho}+\frac{\varrho}{2}, \\
& y_{7} y_{7}^{2} \leq-\frac{y_{7}^{2}}{\tau_{7}}+\frac{M_{7}^{2}\left|y_{7}^{2}\right|}{2 \varrho}+\frac{\varrho}{2}, \\
& y_{9} y_{9}^{2} \leq-\frac{y_{9}^{2}}{\tau_{9}}+\frac{M_{9}^{2}\left|y_{9}^{2}\right|}{2 \varrho}+\frac{\varrho}{2},
\end{aligned}
$$

and using the following inequalities,

$$
\eta_{i} S_{(2 i-1)}\left(S_{2 i}+y_{2 i}\right) \leq \eta_{i}^{2} S_{(2 i-1)}^{2}+\frac{1}{2} S_{2 i}^{2}+\frac{1}{2} y_{2 i}^{2}, \quad i=1, \ldots, 6,
$$

which together with (B.1) implies that

$$
\begin{aligned}
\dot{\Gamma} \leq & \frac{1}{2} \tilde{x}^{T} P \tilde{x}+\frac{1}{2} \tilde{x}^{T} P \widetilde{x}-\sum_{i=1}^{6}\left(c_{(2 i-1)}-\eta_{i}^{2}\right) S_{(2 i-1)}^{2}-\sum_{i=1}^{6}\left(c_{2 i}-1\right) S_{2 i}^{2} \\
& +\sum_{i=1}^{6} \lambda_{i} \tilde{\theta}_{i} \hat{\theta}_{i}+\sum_{i=1}^{6}\left(\frac{1}{2}+\frac{1}{2} \varepsilon_{i}^{2}\right)-\sum_{i=1}^{6}\left(\frac{1}{\tau_{2 i}}-\frac{M_{i}^{2}}{2 \varrho}-\frac{1}{2}\right) y_{2 i}^{2} \\
& -\left(\frac{1}{\tau_{7}}-\frac{M_{7}^{2}}{2 \varrho}\right) y_{7}^{2}-\left(\frac{1}{\tau_{9}}-\frac{M_{9}^{2}}{2 \varrho}\right) y_{9}^{2}+4 \varrho \\
& \leq-\frac{1}{2} \lambda_{\min }\left(Q_{1}\right)\|\widetilde{x}\|^{2}+\tilde{x}^{T} P \sum_{i=1}^{6} b_{i} \widetilde{W}_{i}^{* T} \xi_{i}\left(\widehat{X}_{i}\right) \\
& +\tilde{x}^{T} P \varepsilon-\sum_{i=1}^{6}\left(c_{(2 i-1)}-\eta_{i}^{2}\right) S_{(2 i-1)}^{2}-\sum_{i=1}^{6}\left(c_{2 i}-1\right) S_{2 i}^{2}+\sum_{i=1}^{6} \lambda_{i} \widetilde{\theta}_{i} \widehat{\theta}_{i} \\
& +\sum_{i=1}^{6}\left(\frac{1}{2}+\frac{1}{2} \varepsilon_{i}^{2}\right)-\sum_{i=1}^{6}\left(\frac{1}{\tau_{2 i}}-\frac{M_{i}^{2}}{2 \varrho}-\frac{1}{2}\right) y_{2 i}^{2} \\
& -\left(\frac{1}{\tau_{7}}-\frac{M_{7}^{2}}{2 \varrho}\right) y_{7}^{2}-\left(\frac{1}{\tau_{9}}-\frac{M_{9}^{2}}{2 \varrho}\right) y_{9}^{2}+4 \varrho
\end{aligned}
$$

where $\varepsilon=\left[0, \varepsilon_{1}, 0, \varepsilon_{2}, 0, \varepsilon_{3}, 0, \varepsilon_{4}, 0, \varepsilon_{5}, 0, \varepsilon_{6}\right]^{T}$ and $\lambda_{\min }\left(Q_{1}\right)$ is the smallest eigenvalue of matrix $Q_{1}$. Applying Young's inequality $a b \leq\left(a^{2}+b^{2}\right) / 2$ and the fact $\xi_{i}^{T}\left(\widehat{X}_{i}\right) \xi_{i}\left(X_{i}\right) \leq 1$, the following inequation can be obtained:

$$
\tilde{x}^{T} P \sum_{i=1}^{6} b_{i} \widetilde{W}_{i}^{* T} \xi_{i}\left(\widehat{X}_{i}\right) \leq \frac{1}{2} \lambda_{\max ^{2}}(P)\|\tilde{x}\|^{2}+\frac{1}{2}\left\|\widetilde{W}^{*}\right\|^{2} \leq \frac{1}{2} \lambda_{\max }^{2}(P)\|\tilde{x}\|^{2}+\frac{1}{2} \tilde{\theta},
$$

where $\tilde{\theta}=\tilde{\theta}_{1}+\widetilde{\theta}_{2}+\widetilde{\theta}_{3}+\widetilde{\theta}_{4}+\widetilde{\theta}_{5}+\widetilde{\theta}_{6}$ and $\lambda_{\max }^{2}(P)$ is the largest eigenvalue of matrix $P$.

$$
\tilde{x}^{T} P \varepsilon \leq \frac{1}{2}\|\tilde{x}\|^{2}+\frac{1}{2} \lambda_{\max }^{2}(P)\left\|\varepsilon^{*}\right\|^{2} .
$$

By using Young's inequality, we obtain

$$
2 \widetilde{\theta}_{i} \widehat{\theta}_{i} \leq \theta_{i}^{2}-\widetilde{\theta}_{\grave{i}}^{2}
$$

Substituting (B.6) and (B.7) into (B.5), we have

$$
\begin{aligned}
\dot{\Gamma} \leq & -\mu_{1}\|\widetilde{x}\|^{2}+\frac{1}{2} \widetilde{\theta}+\frac{1}{2} \lambda_{\max ^{2}}(P)\left\|\mathcal{E}^{*}\right\|^{2}-\sum_{i=1}^{6}\left(c_{(2 i-1)}-\eta_{i}^{2}\right) S_{(2 i-1)}^{2} \\
& -\sum_{i=1}^{6}\left(c_{2 i}-1\right) S_{2 i}^{2} \\
& -\sum_{i=1}^{6} \frac{1}{2} \lambda_{i} \widetilde{\theta}_{i}^{2}-\sum_{i=1}^{6}\left(\frac{1}{2}+\frac{1}{2} \varepsilon_{i}^{2}\right)-\sum_{i=1}^{6}\left(\frac{1}{\tau_{2 i}}-\frac{M_{i}^{2}}{2 \varrho}-\frac{1}{2}\right) y_{2 i}^{2} \\
& -\left(\frac{1}{\tau_{7}}-\frac{M_{7}^{2}}{2 \varrho}\right) y_{7}^{2}-\left(\frac{1}{\tau_{9}}-\frac{M_{9}^{2}}{2 \varrho}\right) y_{9}^{2}+4 \varrho+\sum_{i=1}^{6} \frac{1}{2} \lambda_{i} \theta_{i}^{2} \\
& \leq-\mu_{1}\|\tilde{x}\|^{2}-\sum_{i=1}^{6}\left(c{ }_{(2 i-1)}-\eta_{i}^{2}\right) S_{(2 i-1)}^{2}-\sum_{i=1}^{6}\left(c_{2 i}-1\right) S_{2 i}^{2} \\
& -\sum_{i=1}^{6} \frac{1}{2} \lambda_{i} \tilde{\theta}_{i}^{2}+\frac{1}{2} \tilde{\theta} \\
& -\sum_{i=1}^{6}\left(\frac{1}{\tau_{2 i}}-\frac{M_{i}^{2}}{2 \varrho}-\frac{1}{2}\right) y_{2 i}^{2}-\left(\frac{1}{\tau_{7}}-\frac{M_{7}^{2}}{2 \varrho}\right) y_{7}^{2}-\left(\frac{1}{\tau_{9}}-\frac{M_{9}^{2}}{2 \varrho}\right) y_{9}^{2}+C
\end{aligned}
$$

where

$$
\begin{aligned}
& \mu_{1}=\left(\frac{1}{2}\right) \lambda_{\min }\left(Q_{1}\right)-\left(\frac{1}{2}\right) \lambda_{\max }^{2}(P)-1, \\
& C=\frac{1}{2} \lambda_{\max }^{2}(P)\left\|\varepsilon^{*}\right\|^{2}+3+4 \varrho+\sum_{i=1}^{6} \frac{1}{2} \lambda_{i} \theta_{i}^{2}-\sum_{i=1}^{6}\left(\frac{1}{2}+\frac{1}{2} \varepsilon_{i}^{2}\right) .
\end{aligned}
$$

Choose the suitable design parameters to make $\mu_{1}>0$ and satisfy $\sqrt{C / \mu_{1}}<\|\tilde{x}\|$. The following conditions hold:

$$
\begin{aligned}
c_{(2 i-1)} & \geq \eta_{i}^{2}+r, \\
c_{2 i} & \geq 1+r, \\
\frac{1}{\tau_{2 i}} & \geq \frac{1}{2}+\frac{M_{i}^{2}}{2 \varrho}+r, \\
\frac{1}{2} \lambda_{i} & \geq \frac{1}{8}+r, \\
\frac{1}{\tau_{7}} & \geq \frac{M_{7}^{2}}{2 \varrho}+r, \\
\frac{1}{\tau_{9}} & \geq \frac{M_{9}^{2}}{2 \varrho}+r,
\end{aligned}
$$

where $r$ is the positive constant. Thus, we have $\dot{\Gamma} \leq 0$. Hence, all the signals of the closed-loop system are semiglobal bounded. Particularly, the tracking errors of position and attitude angle can converge to an arbitrarily residual set and are always kept in the prespecified cures. This completes the proof. 


\section{Data Availability}

The data used to support the findings of this study are available from the corresponding author upon request.

\section{Conflicts of Interest}

The authors declare that there are no conflicts of interest regarding the publication of this article.

\section{Acknowledgments}

This work was supported in part by the NSF of China under Grant 61673101 and 61304015, the Natural Science Foundation of Jilin Province under Grant 20180201009SF and $20170414011 \mathrm{GH}$, the Thirteenth Five-Year Science Research Plan of Jilin Province under Grant JJKH20200119KJ, and the Jilin Technological Innovation Development Plan under Grant 201831719.

\section{References}

[1] Z. Bo, B. Xian, Z. Yao, and Z. Xu, "Nonlinear robust adaptive tracking control of a quadrotor uav via immersion and invariance methodology," IEEE Transactions on Industrial Electronics, vol. 62, no. 5, pp. 2891-2902, 2015.

[2] P. E. I. Pounds and A. M. Dollar, "Stability of helicopters in compliant contact under PD-PID control," IEEE Transactions on Robotics, vol. 30, no. 6, pp. 1472-1486, 2014.

[3] T. Huang, D. Huang, Z. Wang, and A. Shah, "Robust tracking control of a quadrotor UAV based on adaptive sliding mode controller," Complexity, vol. 2019, Article ID 7931632, 15 pages, 2019.

[4] H. Kong, C. Yang, G. Li, and S.-L. Dai, "A semg-based shared control system with no-target obstacle avoidance for omnidirectional mobile robots," IEEE Access, vol. 8, pp. 2603026040, 2020.

[5] X. Zhang, Y. Wang, G. Zhu et al., "Compound adaptive fuzzy quantized control for quadrotor and its experimental verification," IEEE Transactions on Cybernetics, pp. 1-13, 2020.

[6] M. Taimoor, S. Iqbal, U. I. Bhatti, and A. A. Khan, "Autonomous flight of unmanned aerial vehicle (UAV) by using linear quadratic regulator (LQR)," International Journal of Control and Automation, vol. 8, no. 1, pp. 9-28, 2015.

[7] F. Rinaldi, S. Chiesa, and F. Quagliotti, "Linear quadratic control for quadrotors uavs dynamics and formation flight," Journal of Intelligent and Robotic Systems, vol. 70, no. 1-4, pp. 203-220, 2013.

[8] T. Ryan and H. Jin Kim, "LMI-based gain synthesis for simple robust quadrotor control," IEEE Transactions on Automation Science and Engineering, vol. 10, no. 4, pp. 1173-1178, 2013.

[9] M. Emam and A. Fakharian, "Attitude tracking of quadrotor uav via mixed $\mathrm{H} 2 / \mathrm{H} \infty$ controller: an LMI based approach," in Proceedings of the 2016 24th Mediterranean Conference on Control and Automation (MED), Athens, Greece, June 2016.

[10] J. P. Ortiz, L. I. Minchala, and M. J. Reinoso, "Nonlinear robust $\mathrm{H}$-infinity PID controller for the multivariable system quadrotor," IEEE Latin America Transactions, vol. 14, no. 3, pp. 1176-1183, 2016.

[11] M. Kerma, A. Mokhtari, B. Abdelaziz, and Y. Orlov, "Nonlinear Ho control of a quadrotor (UAV), using high order sliding mode disturbance estimator," International Journal of Control, vol. 85, no. 12, pp. 1876-1885, 2012.
[12] H. Voos, "Nonlinear control of a quadrotor micro-UAV using feedback-linearization," in Proceedings of the IEEE International Conference on Mechatronics, pp. 1-6, Malaga, Spain, April 2009.

[13] K. Alexis, G. Nikolakopoulos, and A. Tzes, "On trajectory tracking model predictive control of an unmanned quadrotor helicopter subject to aerodynamic disturbances," Asian Journal of Control, vol. 16, no. 1, pp. 209-224, 2014.

[14] A. Hao, H. Xia, and C. Wang, "Barrier lyapunov functionbased adaptive control for hypersonic flight vehicles," Nonlinear Dynamics, vol. 88, no. 3, pp. 1833-1853, 2017.

[15] P. Deng, L. Zeng, and Y. Liu, "RBF neural network backstepping sliding mode adaptive control for dynamic pressure cylinder electrohydraulic servo pressure system," Complexity, vol. 2018, Article ID 4159639, 16 pages, 2018.

[16] F. Chen, W. Lei, K. Zhang, G. Tao, and B. Jiang, "A novel nonlinear resilient control for a quadrotor UAV via backstepping control and nonlinear disturbance observer," Nonlinear Dynamics, vol. 85, no. 2, pp. 1281-1295, 2016.

[17] F. Chen, R. Jiang, K. Zhang, B. Jiang, and G. Tao, "Robust backstepping sliding-mode control and observer-based fault estimation for a quadrotor UAV," IEEE Transactions on Industrial Electronics, vol. 63, no. 8, pp. 5044-5056, 2016.

[18] G. Perozzi, D. Efimov, J. M. Biannic, and L. Planckaert, "Trajectory tracking for a quadrotor under wind perturbations: sliding mode control with state-dependent gains," Journal of the Franklin Institute, vol. 355, no. 12, pp. 48094838, 2018.

[19] S. Li, Y. Wang, J. Tan, and Y. Zheng, "Adaptive RBFNNs/ integral sliding mode control for a quadrotor aircraft," Neurocomputing, vol. 216, pp. 126-134, 2016.

[20] Y. Yin, H. Niu, and X. Liu, "Adaptive neural network sliding mode control for quad tilt rotor aircraft," Complexity, vol. 2017, Article ID 7104708, 13 pages, 2017.

[21] W. Hao and B. Xian, "Nonlinear adaptive fault-tolerant control for a quadrotor UAV based on immersion and invariance methodology," Nonlinear Dynamics, vol. 90, no. 4, pp. 2813-2826, 2017.

[22] X. Shao, J. Liu, H. Cao, C. Shen, and H. Wang, "Robust dynamic surface trajectory tracking control for a quadrotor UAV via extended state observer," International Journal of Robust and Nonlinear Control, vol. 28, no. 7, pp. 2700-2719, 2018.

[23] T. Dierks and S. Jagannathan, "Output feedback control of a quadrotor UAV using neural networks," IEEE Transactions on Neural Networks, vol. 21, no. 1, pp. 50-66, 2010.

[24] G. Zhu, L. Nie, Z. Lv, L. Sun, X. Zhang, and C. Wang, "Adaptive fuzzy dynamic surface sliding mode control of large-scale power systems with prescribe output tracking performance," ISA Transactions, vol. 99, pp. 305-321, 2020.

[25] X. Zhang, Y. Wang, X. Chen et al., "Decentralized adaptive neural approximated inverse control for a class of large-scale nonlinear hysteretic systems with time delays," IEEE Transactions on Systems, Man, and Cybernetics: Systems, vol. 49, no. 12, pp. 2424-2437, 2019.

[26] C. Yang, Y. Jiang, J. Na, Z. Li, L. Cheng, and C.-Y. Su, "Finitetime convergence adaptive fuzzy control for dual-arm robot with unknown kinematics and dynamics," IEEE Transactions on Fuzzy Systems, vol. 27, no. 3, pp. 574-588, 2019.

[27] X. Zhang, Z. Xu, C.-Y. Su et al., "Fuzzy approximator based adaptive dynamic surface control for unknown time delay nonlinear systems with input asymmetric hysteresis nonlinearities," IEEE Transactions on Systems, Man, and Cybernetics: Systems, vol. 47, no. 8, pp. 2218-2232, 2017. 
[28] H. M. Lin, T. Dierks, S. Jagannathan, and L. Acar, "Modified consensus-based output feedback control of quadrotor UAV formations using neural networks," Journal of Intelligent and Robotic Systems, vol. 94, no. 1, pp. 283-300, 2019.

[29] Z. Shen, F. Li, X. Cao, and C. Guo, "Prescribed performance dynamic surface control for trajectory tracking of quadrotor uav with uncertainties and input constraints," International Journal of Control, pp. 1-11, 2020.

[30] M. A. M. Basri, A. R. Husain, and K. A. Danapalasingam, "Enhanced backstepping controller design with application to autonomous quadrotor unmanned aerial vehicle," Journal of Intelligent and Robotic Systems, vol. 79, no. 2, pp. 295-321, 2015.

[31] D. Swaroop, J. K. Hedrick, P. P. Yip, and J. C. Gerdes, "Dynamic surface control for a class of nonlinear systems," IEEE Transactions on Automatic Control, vol. 45, no. 10, pp. 1893-1899, 2000.

[32] X. Zhang, Y. Wang, C. Wang, C.-Y. Su, Z. Li, and X. Chen, "Adaptive estimated inverse output-feedback quantized control for piezoelectric positioning stage," IEEE Transactions on Cybernetics, vol. 49, no. 6, pp. 2106-2118, 2019.

[33] X. Zhang, Z. Li, C.-Y. Su, Y. Lin, and Y. Fu, "Implementable adaptive inverse control of hysteretic systems via output feedback with application to piezoelectric positioning stages," IEEE Transactions on Industrial Electronics, vol. 63, no. 9, pp. 5733-5743, 2016.

[34] L.-X. Wang and J. M. Mendel, "Fuzzy basis functions, universal approximation, and orthogonal least-squares learning," IEEE Transactions on Neural Networks, vol. 3, no. 5, pp. 807-814, 1992.

[35] C. Yang, C. Chen, W. He, R. Cui, and Z. Li, "Robot learning system based on adaptive neural control and dynamic movement primitives," IEEE Transactions on Neural Networks and Learning Systems, vol. 30, no. 3, pp. 777-787, 2019.

[36] X. Zhang, B. Li, X. Chen, Z. Li, Y. Peng, and S. Chunyi, "Adaptive implicit inverse control for a class of discrete-time hysteretic nonlinear systems and its application," IEEE Transactions on Mechatronics, 2020.

[37] C. Wang and Y. Lin, "Multivariable adaptive backstepping control: a norm estimation approach," IEEE Transactions on Automatic Control, vol. 57, no. 4, pp. 989-995, 2012.

[38] X. Zhang, C.-Y. Su, Y. Lin, L. Ma, and J. Wang, "Adaptive neural network dynamic surface control for a class of timedelay nonlinear systems with hysteresis inputs and dynamic uncertainties," IEEE Transactions on Neural Networks and Learning Systems, vol. 26, no. 11, pp. 2844-2860, 2015.

[39] Y. Zhou, M. Chen, and C. Jiang, "Robust tracking control of uncertain MIMO nonlinear systems with application to UAVs," IEEE/CAA Journal of Automatica Sinica, vol. 2, no. 1, pp. 25-32, 2015.

[40] H. Wang, B. Su, Y. Wang, and J. Gao, "Adaptive sliding mode fixed-time tracking control based on fixed-time sliding mode disturbance observer with dead-zone input," Complexity, vol. 2019, Article ID 8951382, 14 pages, 2019.

[41] Y. Li, S. Tong, L. Liu, and G. Feng, "Adaptive output-feedback control design with prescribed performance for switched nonlinear systems," Automatica, vol. 80, pp. 225-231, 2017.

[42] Y. Li and S. Tong, "Adaptive fuzzy output-feedback stabilization control for a class of switched nonstrict-feedback nonlinear systems," IEEE Transactions on Cybernetics, vol. 47, no. 4, pp. 1007-1016, 2017.

[43] L. Zhang, Y. Li, and S. Tong, "Adaptive fuzzy output feedback control for MIMO switched nonlinear systems with prescribed performances," Fuzzy Sets and Systems, vol. 306, pp. 153-168, 2017.

[44] L. Xing, C. Wen, Y. Zhu, H. Su, and Z. Liu, "Output feedback control for uncertain nonlinear systems with input quantization," Automatica, vol. 65, pp. 191-202, 2016.

[45] X. Zhang, X. Chen, G. Zhu, and C.-Y. Su, "Output feedback adaptive motion control and its experimental verification for time-delay nonlinear systems with asymmetric hysteresis," IEEE Transactions on Industrial Electronics, vol. 67, no. 8, pp. 6824-6834, 2020.

[46] C. P. Bechlioulis and G. A. Rovithakis, "Adaptive control with guaranteed transient and steady state tracking error bounds for strict feedback systems," Automatica, vol. 45, no. 2, pp. 532-538, 2009.

[47] C. P. Bechlioulis and G. A. Rovithakis, "Prescribed performance adaptive control for multi-input multi-output affine in the control nonlinear systems," IEEE Transactions on Automatic Control, vol. 55, no. 5, pp. 1220-1226, 2010.

[48] Y. H. Kim, F. L. Lewis, and C. T. Abdallah, "A dynamic recurrent neural-network-based adaptive observer for a class of nonlinear systems," Automatica, vol. 33, no. 8, pp. 15391543, 1997.

[49] C.-K. Chan and C.-C. Tsai, "Intelligent backstepping slidingmode control using recurrent interval type 2 fuzzy neural networks for a ball-riding robot," in Proceedings of the 2012 International Conference on Fuzzy Theory and Its Applications (IFUZZY2012), Taichung, Taiwan, November 2012.

[50] Y. Fang, J. Fei, and T. Hu, "Adaptive backstepping fuzzy sliding mode vibration control of flexible structure," Journal of Low Frequency Noise Vibration and Active Control, vol. 37, no. 4, pp. 1079-1096, 2018. 